\title{
CDNA-AFLP analysis reveals the adaptive responses of citrus to long-term boron-toxicity
}

Peng Guo ${ }^{1,2}$, Yi-Ping Qi ${ }^{3}$, Lin-Tong Yang ${ }^{1,2}$, Xin Ye ${ }^{1}$, Huan-Xin Jiang ${ }^{2,4}$, Jing-Hao Huang ${ }^{2,4,5}$ and Li-Song Chen ${ }^{1,2,6,7^{*}}$

\begin{abstract}
Background: Boron (B)-toxicity is an important disorder in agricultural regions across the world. Seedlings of 'Sour pummelo' (Citrus grandis) and 'Xuegan' (Citrus sinensis) were fertigated every other day until drip with $10 \mu \mathrm{M}$ (control) or $400 \mu \mathrm{M}$ (B-toxic) $\mathrm{H}_{3} \mathrm{BO}_{3}$ in a complete nutrient solution for 15 weeks. The aims of this study were to elucidate the adaptive mechanisms of citrus plants to B-toxicity and to identify B-tolerant genes.

Results: B-toxicity-induced changes in seedlings growth, leaf $\mathrm{CO}_{2}$ assimilation, pigments, total soluble protein, malondialdehyde (MDA) and phosphorus were less pronounced in C. sinensis than in C. grandis. B concentration was higher in B-toxic C. sinensis leaves than in B-toxic C. grandis ones. Here we successfully used CDNA-AFLP to isolate 67 up-regulated and 65 down-regulated transcript-derived fragments (TDFs) from B-toxic C. grandis leaves, whilst only 31 up-regulated and 37 down-regulated TDFs from B-toxic C. sinensis ones, demonstrating that gene expression is less affected in B-toxic C. sinensis leaves than in B-toxic C. grandis ones. These differentially expressed TDFs were related to signal transduction, carbohydrate and energy metabolism, nucleic acid metabolism, protein and amino acid metabolism, lipid metabolism, cell wall and cytoskeleton modification, stress responses and cell transport. The higher B-tolerance of $C$. sinensis might be related to the findings that B-toxic C. sinensis leaves had higher expression levels of genes involved in photosynthesis, which might contribute to the higher photosyntheis and light utilization and less excess light energy, and in reactive oxygen species (ROS) scavenging compared to B-toxic C. grandis leaves, thus preventing them from photo-oxidative damage. In addition, B-toxicity-induced alteration in the expression levels of genes encoding inorganic pyrophosphatase 1, AT4G01850 and methionine synthase differed between the two species, which might play a role in the B-tolerance of $C$. sinensis.
\end{abstract}

Conclusions: $C$. sinensis leaves could tolerate higher level of B than C. grandis ones, thus improving the B-tolerance of $C$. sinensis plants. Our findings reveal some novel mechanisms on the tolerance of plants to B-toxicity at the gene expression level.

Keywords: Boron-tolerance, Boron-toxicity, CDNA-AFLP, Citrus grandis, Citrus sinensis, Photosynthesis

\section{Background}

Althought boron (B) is a micronutrient element required for normal growth and development of higher plants, it is harmful to plants when present in excess. Whilst of lesser importance than B-deficiency (a widespread problem in many agricultural crops), B-toxicity is also an important problem in agricultural regions across the world, which citrus trees are cultivated [1-3]. Despite the

\footnotetext{
* Correspondence: lisongchen2002@hotmail.com

${ }^{1}$ College of Resource and Environmental Science, Fujian Agriculture and Forestry University, Fuzhou 350002, China

${ }^{2}$ Institute of Horticultural Plant Physiology, Biochemistry and Molecular Biology, Fujian Agriculture and Forestry University, Fuzhou 350002, China Full list of author information is available at the end of the article
}

importance of B-toxicity for crop productivity, the mechanisms by which plants respond to B-toxicity are poorly understood yet. Recently, increasing attention has been paid to plant B-toxicity as a result of the increased demand for desalinated water, in which the $B$ level may be too high for healthy irrigation of crops [4].

Alteration of gene expression levels is an inevitable process of plants responding to environmental stresses. Kasajima and Fujiwara first investigated high B-induced changes in gene expression in Arabidopsis thaliana roots and rosette leaves using microarray, and identified a number of high B-induced genes, including a heat shock protein and a number of the multi-drug and toxic compound extrusion (MATE) family transporters [5]. Hassan et al. 
preformed suppression subtractive hybridization on root cDNA from bulked B-tolerant and -intolerant doubled haploid barley lines grown under moderate B-stress and identified 111 upregulated clones in the tolerant bulk under B-stress, nine of which were genetically mapped to B-tolerant quantitative trait loci. An antioxidative response mechanism was suggested to provide an advantage in tolerating high level of soil B [6]. Recently, Aquea et al. found that B-toxicity upregulated the expression of genes related to ABA signaling, ABA response and cell wall modification, and downregulated the expression of genes involved in water transporters in Arabidopsis roots, concluding that root growth inhibition was caused by B-toxicity-induced water-stress [7]. Most research, however, has focused on roots and herbaceous plants (i.e., barley, A. thaliana), very little is known about the differential expression of genes in response to B-toxicity in leaves and woody plants.

Citrus belongs to evergreen subtropical fruit trees. In China, B-toxicity often occurs in citrus orchards from high level of B in soils and/or irrigation water and from inappropriate application of B fertilizer especially under low-rainfall conditions [8,9]. During 1998-1999, Huang et al. investigated the nutrient status of soils and leaves from 200 'Guanximiyou' pummelo (Citrus grandis) orchards located in Pinghe, Zhangzhou, China. Up to $61.5 \%$ and $17.0 \%$ of orchards were excess in leaf B and soil water-soluble B, respectively [10]. Previous studies showed that B-toxicity disturbed citrus plant growth and metabolism in multiple way, including interference of nutrient uptake [2], ultrastructural damage of roots and leaves [11-13], inhibition of $\mathrm{CO}_{2}$ assimilation, photosynthetic enzymes and photosynthetic electron transport, decrease of chlorophyll (Chl), carotenoid (Car) and total soluble protein levels, affecting leaf carbohydrate metabolism and antioxidant system $[9,14]$. However, our understanding of the molecular mechanisms underlying these processes in citrus is very limited. To our best knowledge, no high B-toxicity-induced changes in gene expression profiles have been reported in citrus plants to date. Here we investigated the effects of B-toxicity on growth, leaf $\mathrm{CO}_{2}$ assimilation, leaf concentrations of malondialdehyde (MDA), pigments and total soluble protein, root and leaf concentration of $\mathrm{B}$, leaf concentration of phosphorus (P), and leaf gene expression profiles using cDNA-amplified fragment length polymorphism (cDNA-AFLP) in Citrus grandis and Citrus sinensis seedlings differing in B-tolerance [13]. The aims of this study were to elucidate the adaptive mechanisms of citrus plants to B-toxicity and to identify B-tolerant genes.

\section{Results}

Effects of B-toxicity on seedlings growth, B concentration in roots and leaves, and $P$ concentration in leaves

Because B is phloem immobile in citrus plants, B-toxic symptoms first developed in old leaves. The typical visible symptom produced in B-toxic leaves was leaf burn (chlorotic and/or necrotic), which only occurred in C. grandis plants. In the later stages, B-toxic leaves shed premature. By contrast, almost no visible symptoms occurred in C. sinensis plants except for very few plants (Additional file 1).

B-toxicity-induced decreases in root, shoot and whole plant dry weights (DWs) were more pronounced in $C$. grandis than in C. sinensis seedlings (Figure 1A-C). Root DW decreased to a larger extent than shoot DW in response to B-toxicity, and resulted in a decrease in root DW/shoot DW ratio of both C. grandis and C. sinensis seedlings (Figure $1 \mathrm{~A}-\mathrm{B}$ and $\mathrm{D}$ ).

B-toxicity increased B concentration in roots and leaves, especially in leaves and decreased $\mathrm{P}$ concentration in $C$. grandis leaves. No significant differences were found in root and leaf $\mathrm{B}$ concentration and leaf $\mathrm{P}$ concentration between the two species at each given $B$ treatment except that $\mathrm{B}$ concentration was higher in B-toxic $C$. sinensis leaves than in B-toxic C. grandis ones (Figure 2).

\section{Effects of B-toxicity on leaf gas exchange, pigments, total soluble protein and MDA}

B-toxicity-induced decreases in both $\mathrm{CO}_{2}$ assimilation and stomatal conductance were higher in C. grandis than in $C$. sinensis leaves. Intercellular $\mathrm{CO}_{2}$ concentration increased in $C$. grandis leaves, but did not significantly change in $C$. sinensis leaves in response to B-toxicity. $\mathrm{CO}_{2}$ assimilation and stomatal conductance in control leaves did not differ between the two species, but were higher in B-toxic C. sinensis leaves than in B-toxic C. grandis ones. Intercellular $\mathrm{CO}_{2}$ concentration in control leaves was higher in C. sinensis than in C. grandis, but the reverse was the case in B-toxic leaves (Figure 3A-C).

B-toxicity decreased concentrations of $\mathrm{Chl} \mathrm{a}+\mathrm{b}$ and Car and ratio of $\mathrm{Chl} \mathrm{a} / \mathrm{b}$ in $C$. grandis and $C$. sinensis leaves. In control leaves, all the three parameters did not differ between the two species, but $\mathrm{Chl} \mathrm{a}+\mathrm{b}$ and $\mathrm{Car}$ concentrations were higher in B-toxic C. sinensis leaves than in B-toxic C. grandis ones (Figure 3E-G).

Leaf concentrations of total soluble protein and MDA were decreased and increased by B-toxicity in C. grandis leaves, respectively, but were not significantly affected in C. sinensis ones (Figure 3D and $\mathrm{H}$ ).

\section{B-toxicity-induced differentially expressed genes revealed by cDNA-AFLP}

Here we used a total of 256 selective primer combinations to isolate the differentially expressed transcript-derived fragments (TDFs) from B-toxic leaves of two citrus species differing in B-tolerance. A representative picture of a silver-stained cDNA-AFLP gel showing B-toxicity-induced genes in C. grandis and C. sinensis leaves was presented in Additional file 2. As shown in Table 1, a total of 6050 clear 


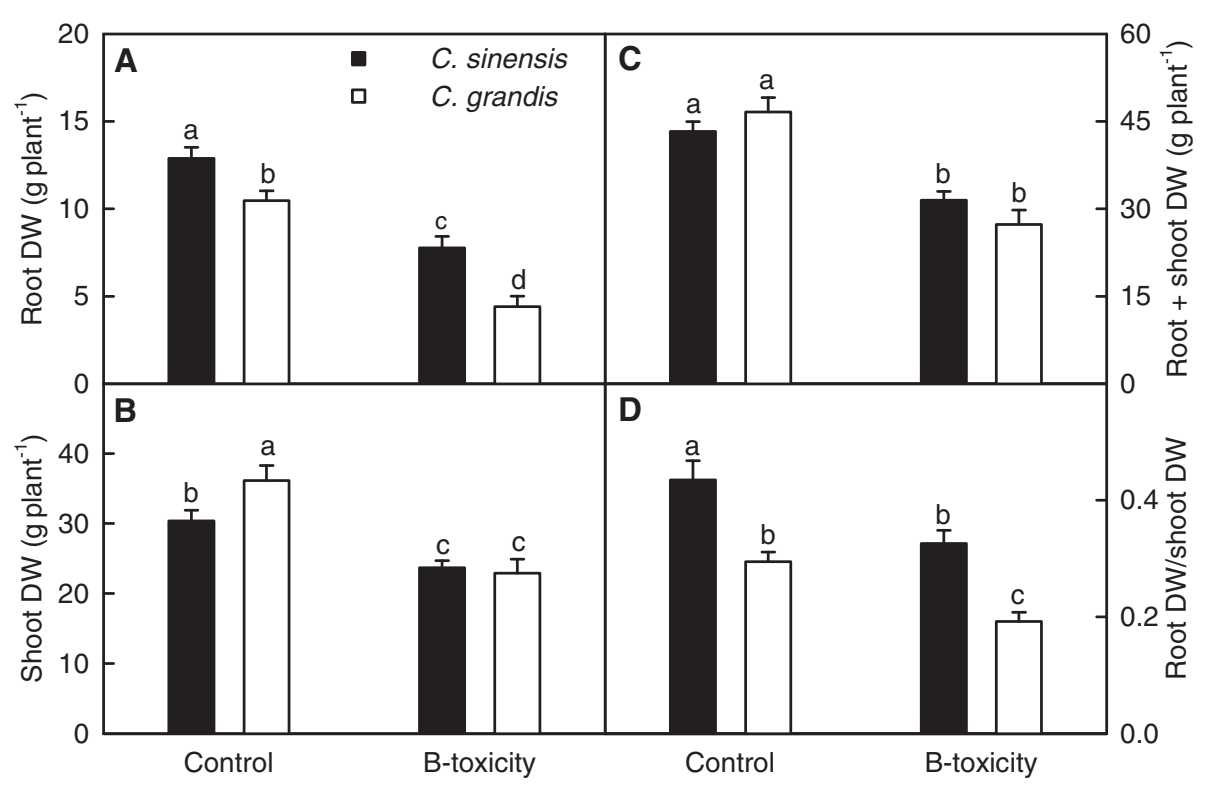

Figure 1 Effects of B-toxicity on growth of Citrus sinensis and C. grandis seedlings. Bars represent means \pm SE $(n=10)$. (A-C) Root, shoot and root + shoot DWs. (D) Ratio of root DW to shoot DW. Bars represent means \pm SE $(n=10)$. Different letters above the bars indicate a significant difference at $P<0.05$.

and unambiguous TDFs were detected from the B-toxic leaves, with an average of 25.7 (15-40) TDFs for each primer combination. Among these TDFs, 932 TDFs only presented in C. grandis, 631 TDFs only presented in $C$. sinensis, and 4587 TDFs presented in the two species.

A total of 218 and 104 differentially expressed and reproducible TDFs were successfully obtained from B-toxic C. grandis and C. sinensis leaves, respectively. All these TDFs were re-amplified, cloned and sequenced. For $C$. grandis, 183 of fragments yielded usable sequence data. Aligment analysis showed $132 \mathrm{TDFs}$ were homologous to genes encoding known, putative predicted, uncharacterized, hypothetical or unnamed proteins, and the remaining 51 TDFs showed no significant matches (Tables 1 and 2). Among these matched TDFs, 67 (50.8\%) TDFs were up-regulated and 65 (49.2\%) were down-regulated by B-toxicity. These TDFs were related to different biological processes such as cell transport (12.9\%), lipid metabolism (2.3\%), nucleic acid metabolism (12.9\%), carbohydrate and energy metabolism (12.1\%), protein and amino acid metabolism (25.0\%), stress responses $(6.1 \%)$, cell wall and cytoskeleton modification (6.8\%), signal transduction (2.3\%), other and unknown processes (19.7\%) (Figure 4A). For C. sinensis leaves, 90 differentially expressed TDFs produced readable sequences (Tables 1 and 2), 68 of which displayed homology to genes encoding known, putative, hypothetical, uncharacterized or unnamed proteins. The remaining 22 TDFs had no database matches. Of these matched TDFs, $31(45.6 \%)$ TDFs increased and 37 (54.4\%) decreased in response to B-toxicity. These TDFs were involved in cell transport (8.8\%), lipid metabolism (4.4\%), nucleic acid metabolism (13.2\%), carbohydrate and energy metabolism (20.6\%), protein and amino acid metabolism (25.0\%), stress responses $(7.4 \%)$, cell wall and cytoskeleton modification (2.9\%), signal transduction (1.5\%), other and unknown processes (16.2\%) (Figure 4B).

\section{Validation of CDNA-AFLP data using qRT-PCR}

In this study, nine TDFs from $C$. sinensis leaves and nine TDFs from C. grandis ones were selected for qRT-PCR analysis in order to validate their expression patterns obtained by cDNA-AFLP analysis. Except for two TDFs (i.e., TDFs \#187_1 and 195_1), the expression profiles of all the TDFs obtained by qRT-PCR were in agreement with the expression patterns produced by cDNA-AFLP (Figure 5). This technique was thus validated in $88.9 \%$ of cases. In addition to gene family complexity, the changes in the intensity of individual bands in the cDNA-AFLP gels might be responsible for the discrepancies between qRT-PCR and CDNA-AFLP analysis.

\section{Discussion}

C. sinensis displayed higher B-tolerance than C. grandis Our results showed that the effects of B-toxicity on plant growth (Figure 1A-C), and leaf gas exchange, pigments, total soluble protein, MDA (Figure 3) and P (Figure 2C) were more pronounced in C. grandis than in C. sinensis seedlings, meaning that $C$. sinensis has higher B-tolerance than $C$. grandis. The present work, like that of the previous workers $[8,13,15]$, indicates that the major of $\mathrm{B}$ in B-toxic citrus plants was accumulated in the 


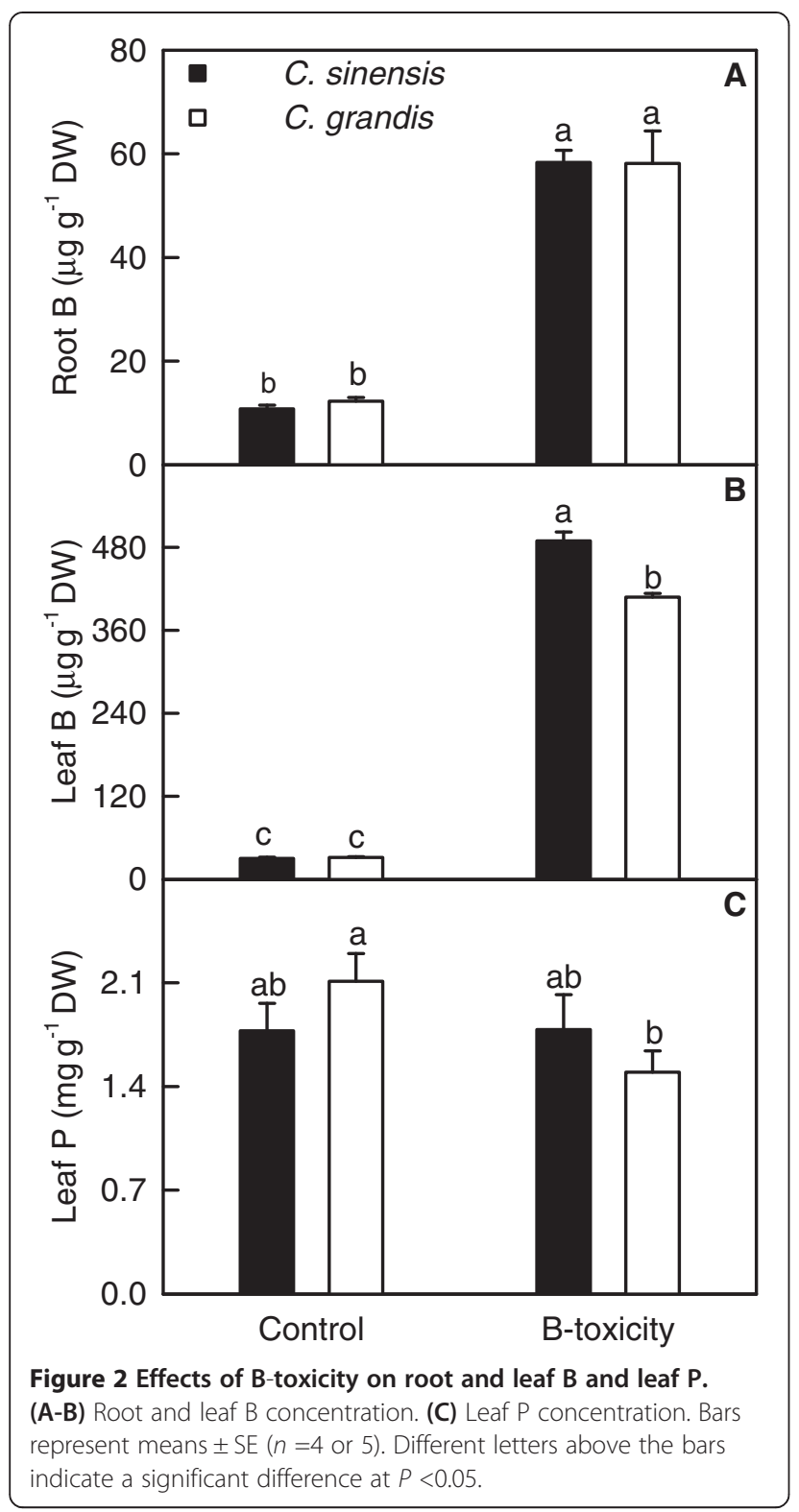

leaves (Figure 2A and B). As shown in Figure 2B, B concentration was not lower in $C$. sinensis than in $C$. grandis leaves regardless of $\mathrm{B}$ concentration in the nutrient solution, indicating that $C$. sinensis leaves may tolerate higher level of B. Similar result has been obtained by Huang et al. [13]. Here we isolated 67 up-regulated and 65 down-regulated TDFs from B-toxic C. grandis leaves, whilst only 31 up-regulated and 37 down-regulated TDFs from B-toxic C. sinensis ones (Figure 4), suggesting that B-toxicity affects $C$. sinensis leaves gene expression less than $C$. grandis ones. These data also support above inference that $C$. sinensis leaves may tolerate higher level of $\mathrm{B}$.

We found that $\mathrm{CO}_{2}$ assimilation was lower in toxic leaves than in control leaves, while stomatal conductance was not lower in the former (Figure 3A-C), implies that $\mathrm{B}$-toxicity-induced inhibition of $\mathrm{CO}_{2}$ assimilation in two citrus species is primarily due to non-stomatal factors. Similar results have been obtained on B-toxic C. grandis and C. sinensis [13,14], 'Navelina' orange and 'Clementine' mandarin plants grafted on sour orange and Swingle citrumelo rootstocks [11,12], Newhall and Skagg's Bonanza navel orange plants grafted on Carrizo citrange and trifoliate orange [9].

\section{Leaf carbohydrate and energy metabolism}

Since B-toxicity decreased $\mathrm{CO}_{2}$ assimilation (Figure 3A), genes involved in photosynthesis and related biological processes might be affected by B-toxicity. As expected, 16 TDFs in C. grandis leaves and 14 TDFs in C. sinensis ones related to carbohydrate and energy metabolism were altered under B-toxicity (Table 2 and Figure 4). We found that B-toxicity decreased the transcript level of ribulose-1,5-bisphosphate (RuBP) carboxylase/oxygenase (Rubisco) small subunit precursor (TDF \#143_2) gene in C. grandis leaves (Table 2), which agrees with the previous report that B-toxicity decreased the activity of Rubisco in C. grandis leaves [14]. Hudson et al. showed that the reduction of Rubisco concentration by anti-small subunit led to decreased photosynthesis in transgenic tobacco plants, but unchanged stomatal conductance [16]. Also, the mRNA abundances of photosystem II (PSII) $32 \mathrm{kDa}$ protein (PsbA, TDF \#251_1), chloroplast PSII oxygen-evolving complex $23 \mathrm{kDa}$ polypeptide (TDF \#112_2) and NifU-like protein (TDF \#239_4) genes were down-regulated in B-toxic C. grandis leaves (Table 2). Khan et al. reported that PsbA knockout tobacco plants lacked PSII activity, accompanied by promoted senescence [17]. By using differential RNA interference (RNAi), Ishihara et al. demonstrated that PSII activity was linearly correlated with the total amount of PsbP (PSII $23 \mathrm{kDa}$ protein) [18]. Ifuku et al. reported that PsbP is essential for the regulation and stabilization of PSII in higher plants [19]. Yabe et al. proposed that Arabidopsis chloroplastic NifU-like protein, which can act as a Fe-S cluster scaffold protein, was required for biogenesis of ferredoxin and photosystem I (PSI) [20]. B-toxicity-induced decreases in the transcript levels of PsbA, chloroplast PSII oxygen-evolving complex $23 \mathrm{kDa}$ polypeptide and NifU-like protein genes agree with our report that B-toxicity impaired the whole photosynthetic electron transport from PSII donor side up to the reduction of end acceptors of PSI in C. grandis leaves [14]. By contrast, B-toxicity increased the transcript levels of chloroplast PSII oxygen-evolving complex $23 \mathrm{kDa}$ polypeptide (TDF \#112_2) and glyceraldehyde-3phosphate dehydrogenase B (TDE \#23_2) in C. sinensis leaves (Table 2). NADP-glyceraldehyde-3-phosphate dehydrogenase is one of the two chloroplast enzymes 


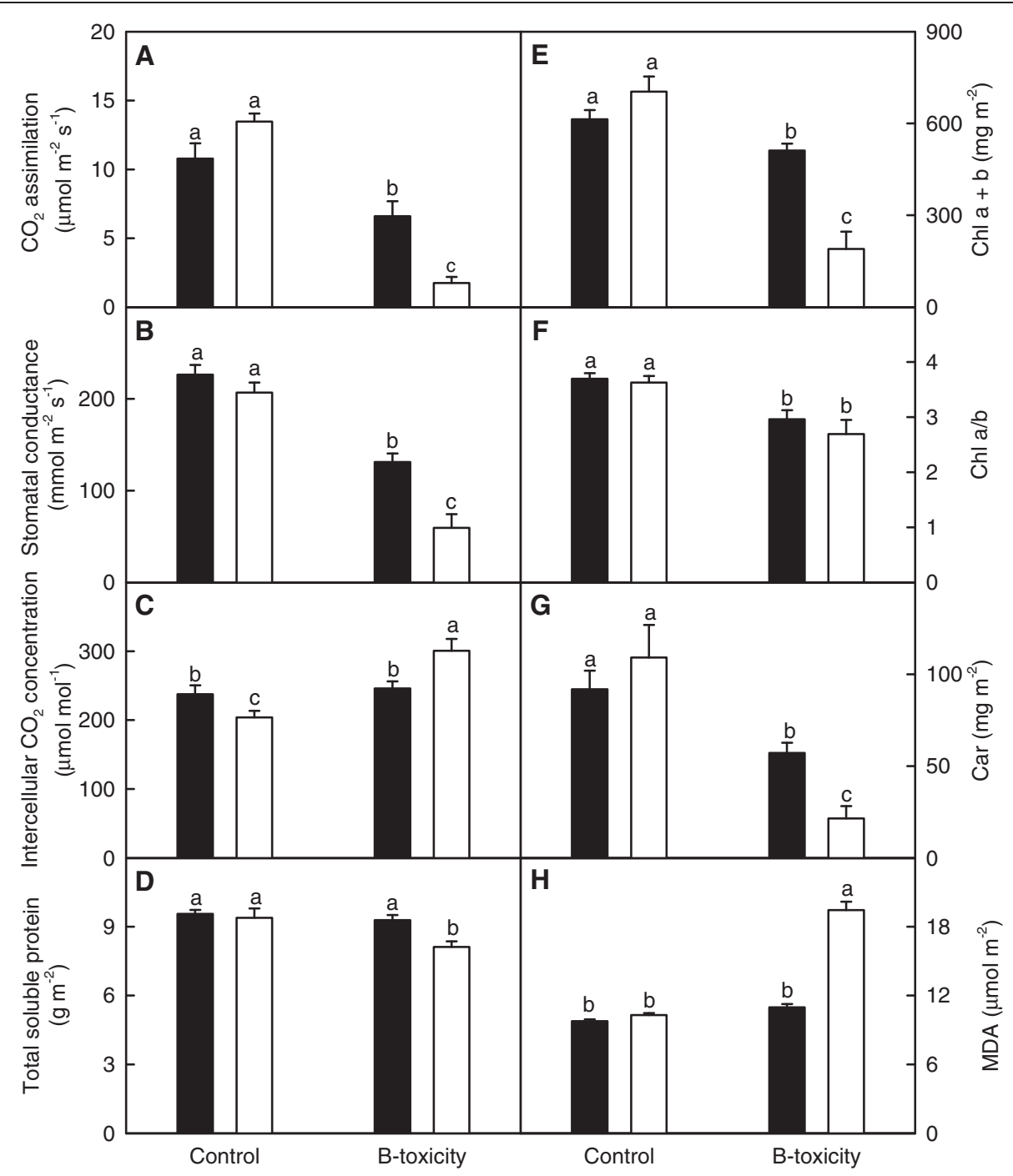

Figure 3 Effects of B-toxicity on leaf gas exchange, total soluble protein, pigments and MDA. (A-C) $\mathrm{CO}_{2}$ assimilation, stomatal conductance and intercellular $\mathrm{CO}_{2}$ concentration. (D) Total soluble protein concentration. (E) $\mathrm{Chl} \mathrm{a}+\mathrm{b}$ concentration. (F) $\mathrm{Chl}$ a/b ratio. (G) Car concentration. (H) MDA concentration. Bars represent means \pm SE ( $n=4$ or 5). Different letters above the bars indicate a significant difference at $P<0.05$.

Table 1 Summary of transcript-derived fragments (TDFs) from control and boron (B)-toxic leaves of Citrus grandis and Citrus sinensis

\begin{tabular}{|c|c|c|c|c|}
\hline & \multicolumn{4}{|l|}{ Number of TDFs } \\
\hline & $\begin{array}{l}\text { Only present in } \\
\text { C. grandis }\end{array}$ & $\begin{array}{l}\text { Only present in } \\
\text { C. sinensis }\end{array}$ & Present in both species & Total \\
\hline Total TDFs detected from gels & 932 & 631 & 4487 & 6050 \\
\hline Total differentially expressed TDFs recovered from gels & 164 & 50 & 54 & 268 \\
\hline TDFs produced useable sequence data & 139 & 46 & 44 & 229 \\
\hline TDFs encoding known or putative proteins & 97 & 40 & 23 & 160 \\
\hline $\begin{array}{l}\text { TDFs encoding predicted, uncharacterized, hypothetical or } \\
\text { unnamed proteins }\end{array}$ & 9 & 2 & 3 & 14 \\
\hline TDFs without database matches & 33 & 4 & 18 & 55 \\
\hline
\end{tabular}


Table 2 Homologies of differentially expressed cDNA-AFLP fragments with known gene sequences in database using BLASTN algorithm along their expression patterns in B-toxic leaves of Citrus grandis and Citrus sinensis

\begin{tabular}{lllcccc}
\hline TDF \# & Size & Homology & Organism origin & E-value & $\begin{array}{l}\text { Similarity } \\
(\%)\end{array}$ & $\begin{array}{l}\text { Genebank ID } \\
\text { (bp) }\end{array}$ \\
& & & $\begin{array}{l}\text { Ratio of } \\
\text { BT/CK }\end{array}$ \\
\cline { 3 - 3 }
\end{tabular}

\section{Carbohydrate and energy metabolism}

251_1 329 Photosystem I| 32 kDa protein (psbA)

112_2 173 Chloroplast photosystem II oxygen-evolving complex $23 \mathrm{kDa}$ polypeptide

239_4 223 NifU-like protein

23_2 253 Glyceraldehyde-3-phosphate dehydrogenase B

6_4 222 Rubisco activase

249_3 313 Sedoheptulose-1 7-bisphosphatase

235_2 305 ADP-glucose pyrophosphorylase

42_1 193 Starch branching enzyme I

59_2 287 Glucose-1-phosphate adenylyltransferase large subunit 1

75_2 $221 \quad$ Citrate synthase

87_1 $224 \quad$ Pyruvate dehydrogenase E1 component subunit beta

33_2 289 Aconitate hydratase 3

161_3 257 2,3-bisphosphoglycerate- independent phosphoglycerate mutase

35_1 $160 \quad$ Plastidial pyruvate kinase 3

130_1 272 Aconitate hydratase 1

171_2 328 Protochlorophyllide oxidoreductase C (PORC, AT1G03630)

5_1 192 Cytochrome P450

76_1 $261 \quad$ Cytochrome P450 like protein

237_2 258 1,3-beta-D-glucanase GH17_65

233_5 $216 \quad$ Alpha-glucan water dikinase 1

57_3 176 UDP-D-glucuronate 4-epimerase 3

117_2 242 Rubredoxin family protein

121_1 179 Rieske iron-sulphur protein precursor

\section{Lipid metabolism}

10_1 282 Fatty acid hydroperoxide lyase

233_3 217 3-oxoacyl-reductase

195_1 $321 \quad$ Sugar-dependent1

8_1 232 Acyl carrier protein 1, chloroplastic-like

194_1 256 Alpha/beta-hydrolase domain-containing protein

186_4 276 Phospholipase-like protein (PEARLI 4) domain-containing protein

\section{Nucleic acid metabolism}

$\begin{array}{lll}52 \_1 & 248 & \text { Spliceosomal protein U1A } \\ 49 \_1 & 337 & \text { Heat stress transcription factor B-2b } \\ 72 \_4 & 171 & \text { Global transcription factor gro + A2 }\end{array}$

Citrus reticulata

Dumortiera hirsuta

Cucumis sativus

Medicago truncatula

Arabidopsis thaliana

A. thaliana

M. truncatula

Pisum sativum

Ipomoea batatas

A. thaliana

Citrus maxima

M. truncatula

Citrus clementina

Vitis amurensis

A. thaliana

Citrus clementina

A. thaliana

Citrus sinensis

A. thaliana

Populus tremula $\times$ Populus tremuloides

A. thaliana

A. thaliana

A. thaliana

Pinellia ternata

\section{Citrus aurantium}

Zea mays

Arabidopsis lyrata

subsp. lyrata

Vitis vinifera

A. thaliana

A. thaliana
A. thaliana
M. truncatula
A. thaliana

$1.00 \mathrm{E}-64 \quad 97 \%$

$1.00 \mathrm{E}-18 \quad 75 \%$

3.00E-17 $\quad 87 \%$

$3.00 \mathrm{E}-06 \quad 84 \%$

$1.00 \mathrm{E}-33 \quad 94 \%$

2.00E-48 97\%

5.00E-39 $82 \%$

$1.00 \mathrm{E}-27 \quad 90 \%$

2.00E-32 $77 \%$

4.00E-34 97\%

$1.00 \mathrm{E}-26 \quad 83 \%$

7.00E-50 94\%

$1.00 \mathrm{E}-40 \quad 91 \%$

$6.00 \mathrm{E}-21 \quad 96 \%$

$2.00 \mathrm{E}-31 \quad 98 \%$

$1.00 \mathrm{E}-43 \quad 89 \%$

$2.00 \mathrm{E}-16 \quad 63 \%$

$3.00 \mathrm{E}-29 \quad 68 \%$

$2.00 \mathrm{E}-31 \quad 78 \%$

4.00E-14 82\%

$1.00 \mathrm{E}-21 \quad 90 \%$

$8.00 \mathrm{E}-24 \quad 81 \%$

$6.00 \mathrm{E}-20 \quad 86 \%$

$2.00 \mathrm{E}-41 \quad 100 \%$

2.00E-05 85\%

$3.00 \mathrm{E}-28 \quad 86 \%$

$6.4 \quad 42 \%$

2.00E-34 72\%

7.00E-10 35\%

1.00E-24 69\%

$8.00 \mathrm{E}-32 \quad 78 \%$

2.00E-04 69\%
AAG49562.1 0

AEI72217.1 0

$\begin{array}{lll}\text { ABK55671.1 } & 0 & 2.9\end{array}$

XP_003594958.1 0

NP_174996.1 +

BAF01986.1 0

XP_003600853.1 + +

CAA69978.1 0

BAE96953.1 0

NP_197423.1 $0 \quad 0$

ADZ05826.1 $\quad 2.8$

XP_003620963.1 +

CBE71057.1 +

ACl96093.1 +

NP_564402.1 0

CBE71056.1 0

$\begin{array}{lll}\text { BAH57125.1 } 0 & 0\end{array}$

AAL24049.1 +

BAE99553.1 +

ADW08745.1 0

NP_563877.1 + 0

$\begin{array}{ll}\text { NP_191922.1 } & 0.3+\end{array}$

NP_568342.1 0

CAM57108.1 +

ABI64149.1 0

NP_001167684.1 0

XP_002871068.1 +

XP_003631979.1 0.4

NP_181474.2 +

NP_973499.1 +

$$
\begin{array}{ll}
\text { NP_182280.1 } & + \\
X_{-} \_003611134.1 & + \\
N P \_192575.3+
\end{array}
$$


Table 2 Homologies of differentially expressed CDNA-AFLP fragments with known gene sequences in database using BLASTN algorithm along their expression patterns in B-toxic leaves of Citrus grandis and Citrus sinensis (Continued)

\begin{tabular}{|c|c|c|c|c|c|c|c|c|}
\hline 120_1 & 257 & IAA13 & Solanum lycopersicum & $3.00 \mathrm{E}-30$ & $67 \%$ & AEX00356.1 & 3.3 & \\
\hline 44_1 & 307 & Elongator complex protein 3 & A. thaliana & $4.00 \mathrm{E}-53$ & $89 \%$ & NP_568725.1 & + & \\
\hline 159_2 & 366 & Flowering time control protein FPA (AT2G43410) & A. thaliana & 0.14 & $32 \%$ & BAH56948.1 & + & \\
\hline 164_1 & 285 & ABA responsive element-binding protein & Solanum torvum & $3.00 \mathrm{E}-10$ & $84 \%$ & AFA37978.1 & + & 0 \\
\hline 73_2 & 255 & Regulator of ribonuclease-like protein & M. truncatula & $2.00 \mathrm{E}-08$ & $83 \%$ & XP_003593378.1 & + & + \\
\hline 250_3 & 305 & RNA recognition motif-containing protein & A. thaliana & 7.00E-31 & $70 \%$ & NP_563946.1 & 0.4 & 2.7 \\
\hline $157 \_2$ & 256 & RNA recognition motif-containing protein & A. thaliana & $3.00 \mathrm{E}-28$ & $76 \%$ & NP_188119.1 & 0 & + \\
\hline $11 \_1$ & 353 & Putative RNA helicase MTR4 & A. thaliana & $1.00 \mathrm{E}-44$ & $82 \%$ & NP_176185.1 & 0 & \\
\hline 71_3 & 209 & RNA helicase SDE3 & A. thaliana & $7.00 \mathrm{E}-24$ & $71 \%$ & AAK40099.1 & 0 & 0 \\
\hline 186_1 & 395 & $\begin{array}{l}\text { Chromodomain-helicase-DNA-binding } \\
\text { protein }\end{array}$ & M. truncatula & $9.00 \mathrm{E}-56$ & $73 \%$ & XP_003625728.1 & & 0 \\
\hline $108 \_1$ & 317 & Receptor for activated $\mathrm{C}$ kinase $1 \mathrm{~B}$ & A. thaliana & $3.00 \mathrm{E}-40$ & $87 \%$ & NP_175296.1 & & 0 \\
\hline $67 \_4$ & 195 & $\begin{array}{l}\text { Sequence-specific DNA binding } \\
\text { transcription factor }\end{array}$ & A. thaliana & 5.2 & $47 \%$ & NP_566386.1 & 0 & \\
\hline $60 \_1$ & 333 & AT5g24120/MLE8_4 & A. thaliana & $9.00 \mathrm{E}-37$ & $63 \%$ & AAK74018.1 & & + \\
\hline $10 \_4$ & 114 & GRAS family transcription factor & Populus trichocarpa & $2.00 \mathrm{E}-04$ & $78 \%$ & XP_002310226.1 & 0 & \\
\hline $22 \_3$ & 248 & MAF1-like protein & Citrus sinensis & $2.00 \mathrm{E}-24$ & $96 \%$ & AEV43358.1 & 0 & \\
\hline $131 \_1$ & 270 & $\begin{array}{l}\text { RNA-binding (RRM/RBD/RNP motifs) family } \\
\text { protein }\end{array}$ & A. thaliana & $7.00 \mathrm{E}-23$ & $62 \%$ & NP_171616.1 & & 7.3 \\
\hline 104_1 & 234 & Zinc finger $\mathrm{CCCH}$ domain-containing protein & M. truncatula & $1.00 \mathrm{E}-04$ & $43 \%$ & XP_003605843.1 & 0 & \\
\hline $68 \_2$ & 217 & F14N23.20 & A. thaliana & $3.00 \mathrm{E}-27$ & $83 \%$ & AAD32882.1 & 0.3 & \\
\hline \multicolumn{9}{|c|}{ Protein and amino acid metabolism } \\
\hline 236_1 & 312 & $\begin{array}{l}\text { Translation initiation factor IF-2, } \\
\text { chloroplastic (AT1G17220) }\end{array}$ & A. thaliana & 4.00E-45 & $85 \%$ & BAH20402.1 & 0 & \\
\hline 117_4 & 174 & Eukaryotic release factor $1-3$ & Brassica oleracea var.botrytis & $3.00 \mathrm{E}-22$ & $94 \%$ & ACZ71035.1 & 0 & \\
\hline $93 \_3$ & 193 & EMB1241 & A.s lyrata subsp. lyrata & $5.00 \mathrm{E}-09$ & $69 \%$ & XP_002873846.1 & 0.4 & \\
\hline $73 \_3$ & 201 & Ankyrin repeat domain-containing protein & M. truncatula & $5.00 \mathrm{E}-19$ & $66 \%$ & XP_003614004.1 & 0.2 & \\
\hline 179_4 & 274 & $50 S$ ribosomal protein L15 & A. thaliana & $1.00 \mathrm{E}-18$ & $80 \%$ & NP_189221.1 & 0 & \\
\hline $105 \_1$ & 216 & $30 \mathrm{~S}$ ribosomal protein S17 & M. truncatula & 0.005 & $89 \%$ & XP_003604547.1 & 0 & 0 \\
\hline $99 \_6$ & 165 & 605 ribosomal protein $L 6$, putative & A. thaliana & $2.00 \mathrm{E}-18$ & $93 \%$ & AAM65875.1 & 0 & \\
\hline $186 \_2$ & 224 & 605 ribosomal protein L4-1 & A. thaliana & $3.00 \mathrm{E}-52$ & $90 \%$ & NP_001030663.1 & 0 & \\
\hline $129 \_2$ & 253 & 605 ribosomal protein $L 23$ & A. thaliana & $2.00 \mathrm{E}-74$ & $97 \%$ & NP_001189805.1 & + & \\
\hline $161 \_1$ & 221 & 605 ribosomal protein $L 10 B$ & Hevea brasiliensis & $3.00 \mathrm{E}-27$ & $83 \%$ & ADR71273.1 & + & \\
\hline 93_2 & 210 & SHEPHERD & A. thaliana & $2.00 \mathrm{E}-26$ & $86 \%$ & BAB86368.1 & + & \\
\hline 98_1 & 272 & Chaperonin 20 & A. thaliana & $2.00 \mathrm{E}-37$ & $81 \%$ & NP_197572.1 & & 0 \\
\hline $69 \_3$ & 174 & AT5G47880 & A. thaliana & $3.00 \mathrm{E}-20$ & $92 \%$ & BAH19602.1 & & 0 \\
\hline 23_4 & 208 & MAP kinase & A. thaliana & $1.00 \mathrm{E}-20$ & $98 \%$ & CAB63149.1 & 0 & \\
\hline 139_4 & 300 & $\begin{array}{l}\text { Putative leucine-rich repeat receptor-like } \\
\text { protein kinase }\end{array}$ & A. thaliana & 4.00E-25 & $55 \%$ & NP_200956.1 & 0 & \\
\hline 72_1 & 238 & CBL-interacting protein kinase 19 & Populus trichocarpa & 8.7 & $89 \%$ & ABJ91226.1 & 0 & \\
\hline 39_3 & 200 & At1g25390/F2J7_14 & A. thaliana & $3.00 \mathrm{E}-23$ & $81 \%$ & AAK97715.1 & 0 & \\
\hline $12 \_2$ & 250 & CDK activating kinase & Nicotiana tabacum & 3.7 & $46 \%$ & BAF75824.1 & + & \\
\hline $22 \_2$ & 252 & Serine/threonine protein kinase ATR & M. truncatula & $6.00 \mathrm{E}-30$ & $83 \%$ & XP_003592675.1 & + & \\
\hline $235 \_3$ & 285 & Receptor-like protein kinase & M. truncatula & $9.00 \mathrm{E}-11$ & $57 \%$ & XP_003621121.1 & + & \\
\hline 110_1 & 408 & Receptor-like protein kinase & A. thaliana & $2.00 \mathrm{E}-31$ & $55 \%$ & BAA96958.1 & & 0 \\
\hline 99_1 & 342 & Protein phosphatase 2C (PP2C) & Fagus sylvatica & $6.00 \mathrm{E}-30$ & $71 \%$ & CAB90633.1 & 2.6 & 3.7 \\
\hline
\end{tabular}


Table 2 Homologies of differentially expressed cDNA-AFLP fragments with known gene sequences in database using BLASTN algorithm along their expression patterns in B-toxic leaves of Citrus grandis and Citrus sinensis (Continued)

\begin{tabular}{|c|c|c|}
\hline $99 \_2$ & 273 & $\mathrm{C} 3 \mathrm{H} 4$ type zinc finger protein \\
\hline 54_1 & 318 & AT5g57360/MSF19_2 \\
\hline 57_1 & 246 & E3 ligase SAP5 \\
\hline 234_1 & 306 & Root phototropism protein 2 \\
\hline 96_1 & 229 & E3 ubiquitin-protein ligase BRE1-like protein \\
\hline 187_1 & 314 & Skp1-like protein 1 \\
\hline 120_2 & 227 & Polyubiquitin \\
\hline $158 \_2$ & 313 & $\begin{array}{l}\text { Putative E3 ubiquitin-protein ligase } \\
\text { XBAT31 isoform } 2\end{array}$ \\
\hline 73_1 & 327 & F-box family protein \\
\hline $112+1$ & 202 & F-box with WD-40 2 \\
\hline 38_3 & 212 & $\begin{array}{l}\text { Drought-inducible cysteine proteinase } \\
\text { RD19A precursor }\end{array}$ \\
\hline $81 \_1$ & 234 & Metalloendopeptidase/zinc ion binding protein \\
\hline 38_4 & 261 & Serine carboxypeptidase II-3 \\
\hline $73 \_4$ & 143 & Proteasome component $(\mathrm{PCl})$ domain protein \\
\hline $240+1$ & 359 & RHOMBOID-like protein 3 \\
\hline 39_1 & 248 & Clp protease proteolytic subunit \\
\hline $145+1$ & 319 & Subtilase family protein \\
\hline $67 \_1$ & 315 & Aminopeptidase family protein \\
\hline $75 \_1$ & 251 & Papain family cysteine protease \\
\hline $138+4$ & 320 & AT4G01850 \\
\hline $245+1$ & 270 & Methionine synthase \\
\hline $231 \_4$ & 216 & $\mathrm{~N}$-carbamoylputrescine amidase \\
\hline $61 \_2$ & 289 & 2-oxoglutarate-dependent dioxygenase \\
\hline 251_3 & 276 & $\begin{array}{l}\text { Cystathionine beta-synthase domain-containing } \\
\text { protein }\end{array}$ \\
\hline
\end{tabular}

A. thaliana
A. thaliana
A. thaliana
A. thaliana
M. truncatula
Prunus avium
Cicer arietinum
Vitis vinifera
Citrus trifoliata
A. thaliana
A. thaliana
A. thaliana
A. thaliana
A. truncatula
A. thaliana
A. thaliana thaliana
Citrus sinensis
A. thaliana
A. thana
A. tha

\begin{tabular}{|c|c|c|c|c|}
\hline 7.00E-28 & $64 \%$ & NP_194986.2 & + & \\
\hline 1.00E-45 & $75 \%$ & AAK64006.1 & + & \\
\hline $2.00 \mathrm{E}-37$ & $84 \%$ & NP_566429.1 & + & \\
\hline $9.00 E-29$ & $60 \%$ & NP_001031446.1 & 2.8 & 3.4 \\
\hline 2.8 & $29 \%$ & XP_003637493.1 & 0 & \\
\hline $4.00 \mathrm{E}-51$ & $85 \%$ & AFJ21662.1 & 0 & \\
\hline 8.00E-39 & $100 \%$ & BAA76429.1 & 0.1 & \\
\hline $2.00 \mathrm{E}-18$ & $63 \%$ & XP_002283974.1 & & + \\
\hline 4.00E-64 & $98 \%$ & ACL51019.1 & & 0 \\
\hline 1.00E-04 & $81 \%$ & NP_567343.1 & & 0 \\
\hline $1.00 \mathrm{E}-15$ & $86 \%$ & BAD94010.1 & 6.0 & 0.3 \\
\hline 1.00E-31 & $84 \%$ & NP_568608.2 & + & \\
\hline 7.00E-21 & $74 \%$ & XP_003589243.1 & 5.9 & \\
\hline 2.00E-07 & $69 \%$ & NP_850994.1 & & + \\
\hline 8.00E-38 & $65 \%$ & NP_196342.1 & & + \\
\hline 2.00E-29 & $100 \%$ & YP_740501.1 & 0 & \\
\hline 3.00E-32 & $62 \%$ & NP_199378.1 & & 0 \\
\hline $2.00 \mathrm{E}-45$ & $85 \%$ & NP_179997.1 & & 0 \\
\hline $3.00 \mathrm{E}-26$ & $85 \%$ & NP_567489.1 & & 0 \\
\hline $3.00 E-59$ & $93 \%$ & BAH20274.1 & & + \\
\hline $2.00 \mathrm{E}-45$ & $98 \%$ & ABS01352.1 & 0 & \\
\hline $6.00 \mathrm{E}-10$ & $76 \%$ & NP_565650.1 & 0.1 & \\
\hline 1.00E-07 & $74 \%$ & XP_002313083.1 & + & \\
\hline 8.00E-45 & $89 \%$ & NP_195409.1 & & 0 \\
\hline
\end{tabular}

\section{Stress responses}

\begin{tabular}{|c|c|c|}
\hline 118_1 & 207 & Inorganic pyrophosphatase 1 \\
\hline 148_2 & 317 & Nudix hydrolase 19 \\
\hline 59_1 & 346 & Fe (II)/ascorbate oxidase family protein SRG1 \\
\hline $137 \_2$ & 156 & Thioredoxin superfamily protein \\
\hline $68 \_3$ & 146 & Thioredoxin superfamily protein \\
\hline 2_1 & 276 & $\begin{array}{l}\text { Group } 5 \text { late embryogenesis abundant } \\
\text { protein (LEA5) }\end{array}$ \\
\hline $125 \_1$ & 389 & Thaumatin-like protein 1 \\
\hline $99 \_5$ & 190 & Protein sodium-and lithium-tolerant 1 \\
\hline 104_3 & 171 & $\begin{array}{l}\text { Transducin/WD40 domain-containing protein } \\
\text { (AtATG18a, AT3G62770) }\end{array}$ \\
\hline $109 \_1$ & 257 & Cold regulated 314 thylakoid membrane 2 \\
\hline 150_2 & 238 & Universal stress protein A-like protein \\
\hline \multicolumn{3}{|c|}{ Signal transduction } \\
\hline 182_2 & 117 & Signal recognition particle 54 kDa protein 2 \\
\hline 108_2 & 257 & 14-3-3 protein \\
\hline 00_1 & 240 & Heterotrimeric GTP-binding protein subunit beta 1 \\
\hline & 252 & Pseudo-response regulator 5 \\
\hline
\end{tabular}
A. thaliana
A. thaliana
A. thaliana
A. thaliana
A. thaliana
Citrus unshiu
Apple tree
A. thaliana
A. thaliana
A. thaliana
M. truncatula

Solanum lycopersicum

Dimocarpus longan

Nicotiana tabacum

Castanea sativa
2.00E-16 83\%

$2.00 \mathrm{E}-48 \quad 78 \%$

2.00E-16 71\%

3.00E-10 58\%

3.00E-07 59\%

$1.00 \mathrm{E}-3594 \%$

$9.00 \mathrm{E}-48 \quad 69 \%$

1.00E-23 92\%

$3.00 \mathrm{E}-20 \quad 94 \%$

1.00E-19 56\%

$4.00 \mathrm{E}-27 \quad 71 \%$

7.00E-07 93\%

6.00E-38 93\%

3.00E-39 94\%

$5.00 \mathrm{E}-12 \quad 86 \%$

$\begin{array}{lll}\text { NP_565052.1 } & 0 & 3.3 \\ \text { NP_197507.1 } & 0 & + \\ \text { NP_173145.1 } & & 0 \\ \text { NP_198706.1 } & + & \\ \text { NP_201385.2 } & 0.1 & \\ \text { ABD93882.1 } & 3.0 & \\ \text { JC7201 } & & \\ \text { NP_973625.1 } & 0 & \\ \text { NP_001030918.4 } & & 0 \\ \text { NP_564327.1 } & & 0 \\ \text { XP_003591417.1 } & 0.2 & \end{array}$

NP_001234428.1 0

ACK76233.1 0

AAG12330.1 0

ABV53464.1 
Table 2 Homologies of differentially expressed CDNA-AFLP fragments with known gene sequences in database using BLASTN algorithm along their expression patterns in B-toxic leaves of Citrus grandis and Citrus sinensis (Continued)

\begin{tabular}{|c|c|c|c|c|c|c|c|c|}
\hline \multicolumn{9}{|c|}{ Cell transport } \\
\hline 26_1 & 342 & $\mathrm{H}^{+}$-ATPase 6, plasma membrane-type & A. thaliana & $1.00 \mathrm{E}-38$ & $97 \%$ & NP_178762.1 & + & \\
\hline 124_3 & 166 & $\begin{array}{l}\text { Calcium-transporting ATPase 1, endoplasmic } \\
\text { reticulum-type (ECA1) }\end{array}$ & A. thaliana & $2.00 \mathrm{E}-14$ & $83 \%$ & NP_172259.1 & 3.1 & \\
\hline 66_1 & 177 & Heavy metal ATPase & P. trichocarpa & $4.00 \mathrm{E}-15$ & $78 \%$ & XP_002303580.1 & + & \\
\hline 97_1 & 201 & Proton pump-interactor 1 (PPI1, AT4G27500) & A. thaliana & $3.00 \mathrm{E}-12$ & $56 \%$ & BAH19433.1 & + & \\
\hline 53_1 & 340 & ABC transporter $\mathrm{G}$ family member 40 & A. thaliana & $9.00 \mathrm{E}-35$ & $67 \%$ & NP_173005.1 & + & \\
\hline 210_1 & 247 & Copper transporter & P. trichocarpa & $2.00 \mathrm{E}-15$ & $64 \%$ & XP_002298334.1 & + & \\
\hline 178_1 & 297 & Cyclic nucleotide-gated ion channel 1 & A. thaliana & 0.002 & $50 \%$ & NP_200125.1 & + & \\
\hline 49_3 & 252 & Vacuolar-sorting receptor 3 & A. thaliana & $1.00 \mathrm{E}-40$ & $77 \%$ & NP_179081.1 & + & \\
\hline 137_1 & 249 & Vacuolar protein-sorting-associated protein 37-1 & A. thaliana & 0.48 & $63 \%$ & NP_190880.1 & + & \\
\hline 63_1 & 357 & $\begin{array}{l}\text { Vesicle-associated membrane protein-associated } \\
\text { protein }\end{array}$ & M. truncatula & $3.00 \mathrm{E}-05$ & $70 \%$ & XP_003608721.1 & + & \\
\hline 51_1 & 316 & SecY protein transport family protein & A. thaliana & $2.00 \mathrm{E}-51$ & $87 \%$ & NP_174225.2 & + & \\
\hline 250_2 & 263 & Fat-free-like protein & M. truncatula & $1.00 \mathrm{E}-32$ & $82 \%$ & XP_003591407.1 & + & \\
\hline $79 \_2$ & 237 & Non-specific lipid-transfer protein & M. truncatula & $1.00 \mathrm{E}-04$ & $53 \%$ & XP_003610781.1 & 2.5 & \\
\hline $67 \_3$ & 268 & Sieve element occlusion protein 1 & Nicotiana tabacum & $6.00 \mathrm{E}-23$ & $65 \%$ & AFN06072.1 & + & + \\
\hline 89_2 & 230 & AT5g24810/F6A4_20 & A. thaliana & $1.00 \mathrm{E}-04$ & $75 \%$ & AAK82520.1 & 0 & \\
\hline $6 \_1$ & 368 & Protein transport protein SEC61 gamma subunit & Zea mays & $2.00 \mathrm{E}-04$ & $92 \%$ & NP_001150911.1 & 0 & \\
\hline $249 \_2$ & 370 & $\begin{array}{l}\text { Putative beta-subunit of adaptor protein } \\
\text { complex } 3 \text {, PAT2 }\end{array}$ & A. thaliana & $2.00 \mathrm{E}-15$ & $42 \%$ & NP_567022.1 & 0 & 0 \\
\hline 61_1 & 228 & Sugar transporter ERD6-like 5 & A. thaliana & 7.00E-15 & $57 \%$ & NP_564665.3 & & 0 \\
\hline 179_2 & 225 & Metal tolerance protein & P. trichocarpa & $6.00 \mathrm{E}-26$ & $70 \%$ & XP_002312066.1 & & 0 \\
\hline 51_4 & 221 & Kinesin-related protein & M. truncatula & 0.38 & $35 \%$ & XP_003612133.1 & & + \\
\hline 36_2 & 319 & Bidirectional sugar transporter SWEET7 & A. thaliana & 5.00E-08 & $60 \%$ & NP_567366.1 & & + \\
\hline \multicolumn{9}{|c|}{ Cell wall and cytoskeleton modification } \\
\hline 49_4 & 210 & Caffeic acid 3-O-methyltransferase & M. truncatula & $9.00 \mathrm{E}-23$ & $68 \%$ & XP_003602597.1 & & 0 \\
\hline $125 \_2$ & 145 & Caffeic acid O-methyltransferase 3 & Gossypium hirsutum & $2.00 \mathrm{E}-05$ & $55 \%$ & ACZ06242.1 & 0.2 & \\
\hline $10 \_3$ & 274 & Chitinase & Citrus sinensis & $3.00 \mathrm{E}-54$ & $94 \%$ & CAA93847.1 & 0 & 0 \\
\hline $249 \_4$ & 217 & Cellulose synthase & Populus tremuloides & $1.00 \mathrm{E}-20$ & $83 \%$ & AAO25581.1 & 0.2 & \\
\hline 33_3 & 249 & O-methyltransferase 1 & A. thaliana & $1.00 \mathrm{E}-33$ & $74 \%$ & AAB96879.1 & + & \\
\hline $241 \_1$ & 326 & LIM domain-containing protein & A. thaliana & $1.00 \mathrm{E}-64$ & $94 \%$ & NP_195404.6 & + & \\
\hline 124_2 & 385 & UDP-glucose flavonoid 7-O-glucosyltransferase & M. truncatula & 4.00E-12 & $73 \%$ & XP_003629628.1 & + & \\
\hline $3 \_3$ & 225 & UDP-glucosyltransferase family 1 protein & Citrus sinensis & $6.00 \mathrm{E}-36$ & $96 \%$ & ACS87993.1 & + & \\
\hline 70_4 & 176 & Limonoid UDP-glucosyltransferase & Citrus sinensis & $2.00 \mathrm{E}-26$ & $98 \%$ & ACD14147.1 & + & \\
\hline 63_2 & 228 & Putative glucosyltransferase & A. thaliana & $2.00 \mathrm{E}-20$ & $63 \%$ & AAM61749.1 & 3.9 & \\
\hline \multicolumn{9}{|c|}{ Other and unknown processes } \\
\hline 229 & 181 & Phytoene synthase & Citrus unshiu & $1.00 \mathrm{E}-26$ & $95 \%$ & AAF33237.1 & 0 & \\
\hline $231 \_1$ & 316 & Strictosidine synthase family protein & A. thaliana & 2.00E-28 & $68 \%$ & NP_191262.2 & 0.4 & 2.6 \\
\hline $72 \_3$ & 194 & $\begin{array}{l}\text { Calcium-dependent lipid-binding } \\
\text { domain-containing protein }\end{array}$ & A. thaliana & 8.00E-19 & $78 \%$ & NP_564576.1 & + & + \\
\hline 135_2 & 335 & Oxidoreductase family protein & $\begin{array}{l}\text { Arabidopsis lyrata } \\
\text { subsp.lyrata }\end{array}$ & $3.00 \mathrm{E}-40$ & $65 \%$ & XP_002874584.1 & & 0 \\
\hline 5_2 & 262 & Alkaline-phosphatase-like protein & A. thaliana & $7.00 \mathrm{E}-44$ & $89 \%$ & NP_194697.1 & 0 & \\
\hline $10 \_5$ & 147 & Protein tolB & M. truncatula & $2.00 \mathrm{E}-06$ & $55 \%$ & XP_003630471.1 & + & \\
\hline
\end{tabular}


Table 2 Homologies of differentially expressed cDNA-AFLP fragments with known gene sequences in database using BLASTN algorithm along their expression patterns in B-toxic leaves of Citrus grandis and Citrus sinensis (Continued)

\begin{tabular}{|c|c|c|c|c|c|c|c|c|}
\hline $231 \_2$ & 285 & $\begin{array}{l}\text { Cofactor of nitrate reductase and xanthine } \\
\text { dehydrogenase } 3\end{array}$ & A. thaliana & $5.00 \mathrm{E}-35$ & $83 \%$ & NP_171636.1 & & 3.9 \\
\hline $51 \_3$ & 256 & Neutral/alkaline non-lysosomal ceramidase & A. thaliana & $3.00 \mathrm{E}-14$ & $71 \%$ & NP_172218.1 & & 0.5 \\
\hline $229 \_2$ & 207 & PQ-loop repeat family protein & A. lyrata subsp. lyrata & $2.00 \mathrm{E}-22$ & $74 \%$ & XP_002870687.1 & + & \\
\hline $71 \_4$ & 206 & Metallo-beta-lactamase domain-containing protein & A. thaliana & $9.00 \mathrm{E}-19$ & $66 \%$ & NP_564334.1 & + & \\
\hline 117_3 & 214 & $\begin{array}{l}\text { Oligosaccharyltransferase complex/magnesium } \\
\text { transporter family protein }\end{array}$ & A. thaliana & $5.00 \mathrm{E}-17$ & $60 \%$ & NP_176372.1 & 0 & \\
\hline $146 \_3$ & 337 & Mitochondrial protein, putative & M. truncatula & $1.00 \mathrm{E}-24$ & $74 \%$ & XP_003588355.1 & 0.4 & 0.3 \\
\hline 20_1 & 287 & AT1G16560 & A. thaliana & $2.00 \mathrm{E}-42$ & $74 \%$ & BAH19866.1 & + & \\
\hline 117_1 & 338 & At2g27385 & A. lyrata subsp. lyrata & $8.00 \mathrm{E}-15$ & $91 \%$ & XP_002880912.1 & 0.2 & \\
\hline 173_1 & 290 & SOUL heme-binding protein & A. thaliana & $1.00 \mathrm{E}-40$ & $90 \%$ & NP_197514.2 & 0 & \\
\hline $122 \_1$ & 166 & AT-LS1 product & A. thaliana & $2.00 \mathrm{E}-21$ & $86 \%$ & CAA41632.1 & 0 & \\
\hline $77 \_2$ & 231 & Alpha/beta-hydrolase family protein & A. thaliana & $3.00 \mathrm{E}-36$ & $94 \%$ & NP_196943.1 & 1.8 & \\
\hline $99 \_3$ & 265 & Conserved hypothetical protein & Ricinus communis & 0.069 & $44 \%$ & XP_002511001.1 & 0 & + \\
\hline $229+1$ & 271 & Conserved hypothetical protein & R. communis & 2.00E-09 & $90 \%$ & XP_002532497.1 & 0 & \\
\hline $70 \_1$ & 267 & Predicted protein & $\begin{array}{l}\text { Micromonas pusilla } \\
\text { CCMP1545 }\end{array}$ & $3.00 \mathrm{E}-49$ & $94 \%$ & XP_003064993.1 & + & + \\
\hline 123 & 364 & PREDICTED: exportin-4-like & Vitis vinifera & $9.00 \mathrm{E}-47$ & $81 \%$ & XP_002266608.2 & + & \\
\hline 23_1 & 308 & Predicted protein & P. trichocarpa & 0.062 & $34 \%$ & XP_002317402.1 & + & \\
\hline 232_2 & 246 & Predicted protein & P. trichocarpa & $5.00 \mathrm{E}-12$ & $48 \%$ & XP_002319603.1 & 0.1 & \\
\hline 237_1 & 265 & $\begin{array}{l}\text { PREDICTED: uncharacterized protein } \\
\text { LOC100776190 }\end{array}$ & Glycine max & 5.8 & $36 \%$ & XP_003524378.1 & 0 & \\
\hline 242_1 & 245 & $\begin{array}{l}\text { PREDICTED: uncharacterized protein } \\
\text { LOC100789831 }\end{array}$ & G. $\max$ & $2.00 \mathrm{E}-07$ & $60 \%$ & XP_003520084.1 & + & \\
\hline 69_2 & 244 & $\begin{array}{l}\text { PREDICTED: uncharacterized protein } \\
\text { LOC100853355 }\end{array}$ & Vitis vinifera & 0.008 & $49 \%$ & XP_003634177.1 & 0 & \\
\hline 130_2 & 210 & Uncharacterized protein & A. thaliana & $6.00 \mathrm{E}-21$ & $79 \%$ & NP_176682.1 & & 1.7 \\
\hline 252_1 & 301 & Uncharacterized protein & A. thaliana & $8.00 \mathrm{E}-16$ & $56 \%$ & NP_001031080.1 & 0 & \\
\hline $97 \_2$ & 163 & Unnamed protein product & Vitis vinifera & 0.079 & $42 \%$ & CBI21631.3 & 0 & 7.0 \\
\hline 91_2 & 270 & Hypothetical protein & A. thaliana & 0.19 & $54 \%$ & AAD21766.1 & & 3.9 \\
\hline $9 \_1$ & 255 & Hypothetical protein MTR_5g051130 & M. truncatula & $1.00 \mathrm{E}-11$ & $100 \%$ & XP_003614394.1 & + & \\
\hline
\end{tabular}

Expression ratio: 0 means TDFs were only detected in control leaves; + means TDF were only detected in the B-toxic leaves. \#: Number; BT: B-toxicity; CK: Control; CG: C. grandis; CS: C. sinensis. Functional classification was performed based on the information reported for each sequence by The Gene Ontology (http://amigo1.geneontology. org/cgi-bin/amigo/blast.cgi) and Uniprot (http://www.uniprot.org/). Relative expression ratio was obtained by gel image analysis, which was performed with PDQuest version 8.0.1 (Bio-Rad, Hercules, CA, USA).

which catalyze the reduction of 3-phosphoglycerate to triose phosphate [21]. However, the expression of Rubisco activase (TDF \#6_4) gene in C. sinensis leaves decreased in response to B-toxicity (Table 2). Generally speaking, B-toxic C. sinensis leaves had higher expression levels of photosynthetic genes than B-toxic C. grandis ones. This might be responsible for the greater decrease in $\mathrm{CO}_{2}$ assimilation in B-toxic C. grandis leaves compared with B-toxic $C$. sinensis ones. It is noteworthy that the mRNA level of gene encoding sedoheptulose-1,7bisphosphatase (SBPase, TDF \#249_3), a key factor for the $\mathrm{RuBP}$ regeneration, was up-regulated in B-toxic leaves of the two citrus species (Table 2). Harrison et al. showed that a small decrease in SBPase activity caused a decline in $\mathrm{CO}_{2}$ assimilation by reducing the capacity for $\mathrm{RuBP}$ regeneration [22]. Lefebvre et al. observed that transgenic tobacco plants over-expressing SBPase had enhanced photosynthesis and growth from an early stage in development [23]. Wang reported that transgenic tomato plants over-expressing SBPase were more tolerance to low temperature and had higher photosynthetic capacity under low temperature [24]. Therefore, the up-regulation of SBPase might be an adaptive response to B-toxicity.

As shown in Table 2, B-toxicity decreased leaf expression levels of three genes [i.e., ADP-glucose pyrophosphorylase (TDF \#235_2) in C. sinensis, starch branching enzyme I 


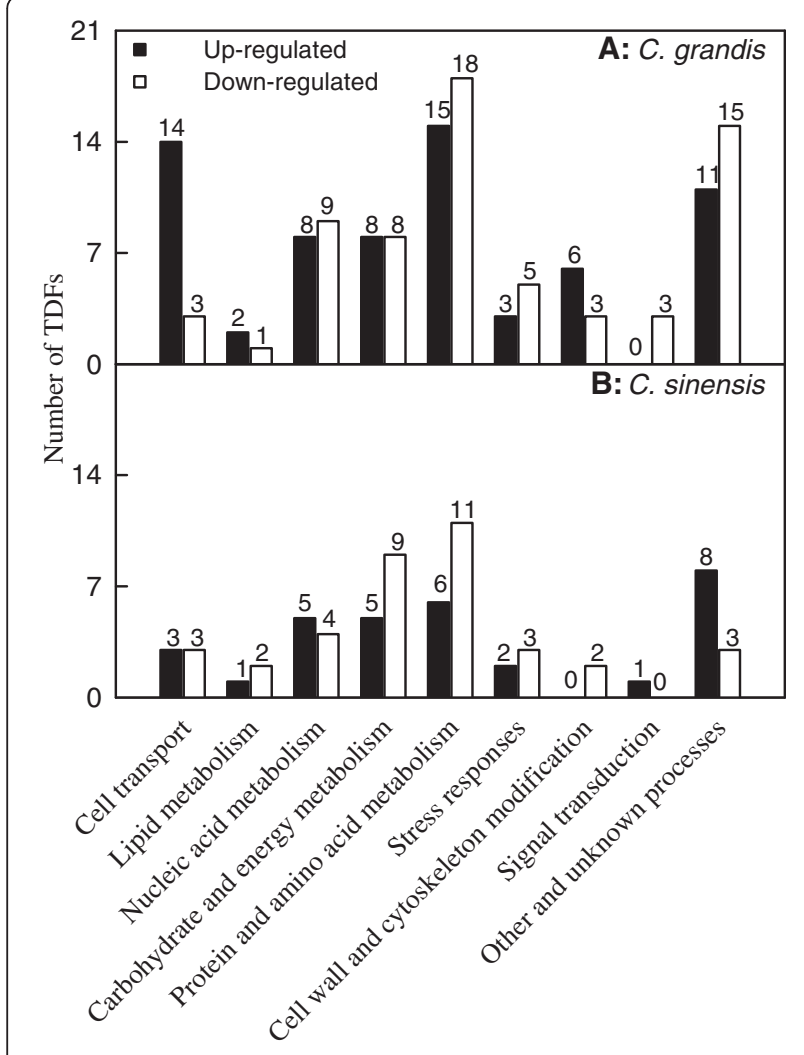

Figure 4 Functional classification of differentially expressed TDFs under B-toxicity in Citrus grandis (A) and Citrus sinensis leaves (B). Functional classification was performed based on the information reported for each sequence by The Gene Ontology (http://amigol.geneontology.org/cgi-bin/amigo/blast.cgi) and Uniprot (http://www.uniprot.org/).

(TDF \#42_1) in C. grandis and glucose-1-phosphate adenylyltransferase large subunit 1 (TDF \#59_2) in the two citrus species] related to starch biosynthesis, which agrees with the previous report that B-toxicity decreased starch concentration in C. grandis leaves [14].

B-toxicity increased the mRNA levels of three genes encoding citrate synthase (TDF \#75-2), pyruvate dehydrogenase E1 component subunit beta (TDF \#87_1) and aconitate hydratase 3 (TDF \#33-2) in C. grandis leaves (Table 2), indicating that tricarboxylic acid cycle might be up-regulated in B-toxic C. grandis leaves. Similarly, the transcript level of a glycolysis gene encoding 2,3-bisphosphoglycerate-independent phosphoglycerate mutase (TDF \#161_3) was enhanced in B-toxic C. sinensis leaves (Table 2). However, the mRNA levels of plastidial pyruvate kinase 3 (TDF \#35_1) and aconitate hydratase 1 (TDF \#33_2) genes were reduced in B-toxic C. sinensis leaves (Table 2). There is evidence showing that plastidic pyruvate kinase plays a key role in fatty acid synthesis by controlling the supply of ATP and pyruvate for de novo fatty acid synthesis in plastids [25]. Thus, the fatty acid metabolism in B-toxic $C$. sinensis leaves might be impaired due to decreased plastidic pyruvate kinase.

In Arabidopsis, three NADPH: protochlorophyllide oxidoreductases (PORs), denoted as PORA, PORB, and PORC participate in mediating the light-dependent protochlorophyllide reduction [26]. Pattanayak and Tripathy showed that over-expression of PORC in Arabidopsis led to coordinated up-regulation of gene/ protein expression of several Chl biosynthetic pathway enzymes, thus enhancing $\mathrm{Chl}$ synthesis, and that the ${ }^{1} \mathrm{O}_{2}$-mediated photo-oxidative damage in transgenic plants overexpressing $P O R C$ was minimal under high light stress [27]. The observed lower transcript level of PORC (TDF \#171_2) in B-toxic C. grandis and $C$. sinensis leaves (Table 2) agrees with the results that B-toxicity decreased the concentration of $\mathrm{Chl} \mathrm{a}+\mathrm{b}$ in citrus leaves (Figure 3E).

Cytochrome P450s play a key role in biotic and abiotic stresses. Transgenic tobacco and potato plants expressing cytochrome P450 with increased monooxygenase activity tolerated better oxidative stress after herbicide treatment [28]. We found that B-toxicity increased the expression levels of genes encoding cytochrome P450 (TDF \#5_1) and cytochrome P450 like protein (TDF \#76-1) in C. grandis leaves (Table 2), which agrees with the previous report that some of the 49 cytochrome P450 genes in Arabidopsis were upregulated by biotic (i.e., Alternaria brassicicola and Alternaria alternata) and abiotic [i.e., drought, high salinity, low temperature, hormones, paraquat, rose bengal, UV stress (UV-C), mechanical wounding and heavy metal stress $\left.\left(\mathrm{CuSO}_{4}\right)\right]$ stresses [29]. Thus, the up-regulation of cytochrome P450s in B-toxic C. grandis leaves might be an adaptive response. However, B-toxicity decreased the expression of cytochrome P450 in Arabidopsis roots [7].

Taken all together, we isolated eight up-regulated and eight down-regulated TDFs from B-toxic C. grandis leaves, and five up-regulated and nine down-regulated from B-toxic C. sinesnsis ones. Among these differentially expressed TDFs, only SBPase (TDF \#249_3) and PORC (TDF \#171_2) were similarly affected by B-toxicity in the two species (Table 2). These results demonstrated that the transcript profiles in the two species were differentially altered under B-toxicity.

\section{Leaf lipid metabolism}

Allene oxide synthase (AOS) and hydroperoxide lyase (HPL) branches of the oxylipin pathway, which are responsible for the production of jasmonates and aldehydes, respectively, participate in a range of stresses. Recently, Liu et al. showed that depletion of rice OsHPL3 greatly stimulated the jasmonic acid-governed defense response [30]. Therefore, the AOS pathway and jasmonate level might be up-regulated in the B-toxic C. sinensis 

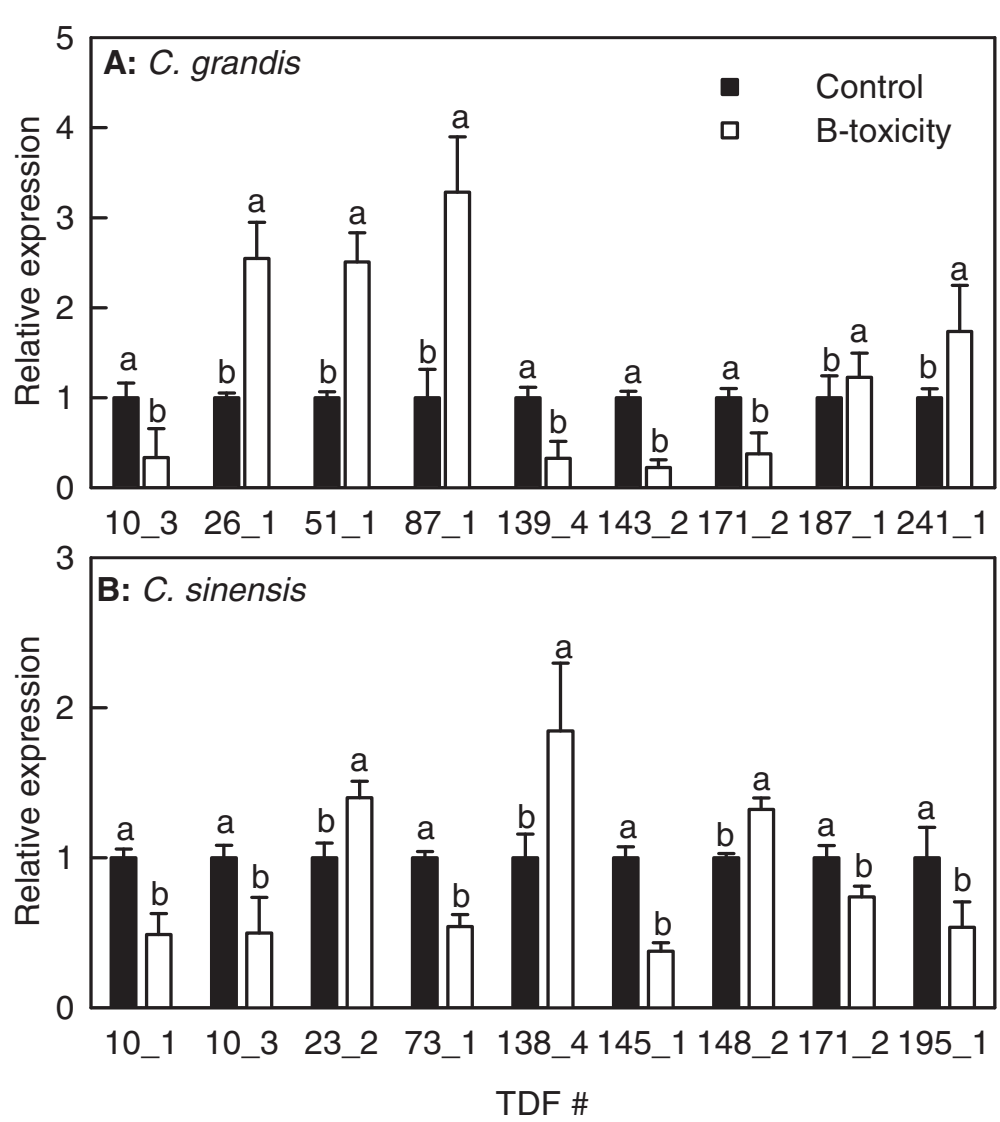

Figure 5 Effects of B-toxicity on gene expression of Citrus grandis (A) and Citrus sinensis (B) leaves. (A) Relative expression levels of genes encoding chitinase (TDF \#10_3), $H^{+}$-ATPase 6 (TDF \#26-1), secY protein transport family protein (TDF \#51_1), pyruvate dehydrogenase E1 component subunit $\beta$ (TDF \#87-1), putative leucine-rich repeat receptor-like protein kinase (TDF \#139_4), Rubisco small subunit precursor (TDF \#143-2), PORC (TDF \#171_2), Skp1-like protein 1 (TDF \#187_1) and LIM domain-containing protein (TDF \#241_1). (B) Relative expression levels of genes encoding fatty acid hydroperoxide lyase (TDF \#10_1), chitinase (TDF \#10_3), glyceraldehyde-3-phosphate dehydrogenase B (TDF \#23-2), F-box family protein (TDF \#73-1), AT4G01850 (TDF \#138_4), subtilase family protein (TDF \#145_1), Nudix hydrolase 19 (TDF \#148_2), PORC (TDF \#171_2) and sugar-dependent1 (TDF \#195_1). Bars represent means \pm SE $(n=3)$. Different letters above the bars indicate a significant difference at $P<0.05$.

leaves due to decreased expression of fatty acid HPL (TDF \#10_1; Table 2), thus contributing to B-tolerance. In addition, B-toxicity also affected the transcript levels of three genes [i.e., plastidial pyruvate kinase 3 (TDF \#35_1), sugar-dependent1 (TDF \#195_1) and 3-oxoacyl-reductase (TDF \#233_3)] related to lipid metabolism in C. sinensis leaves (Table 2). Thus, lipid metabolism might be altered in B-toxic C. sinensis leaves.

Tang et al. reported that transgenic tobacco plants over-expressing acyl carrier protein $(A C P)-1$ (or expressing antisense $A C P 1$ ) exhibited an increase (or decrease) in leaf concentrations of total lipids and the main fatty acids, and were more tolerant (or sensitive) to cold stress [31]. Branen et al. showed that reduction of ACP4 by antisense RNA led to a decrease in total leaf lipids and decreased photosynthetic efficiency, and concluded that $A C P 4$ might play a major role in the biosynthesis of fatty acids for chloroplast membrane development [32]. The lower transcript level of gene encoding
ACP1, chloroplastic-like (TDF \#8_1) in B-toxic C. grandis leaves (Table 2) means that fatty acid biosynthesis in these leaves might be impaired. However, the expression of $\alpha / \beta$-hydrolase domain-containing protein (TDF \#194_1) and phospholipase-like protein (PEARLI 4) domain-containing protein (TDF \#186_4) genes were up-regulated in B-toxic C. grandis leaves (Table 2).

\section{Leaf nucleic acid metabolism}

As shown in Table 2, eight up-regulated genes (TDFs \#52_1, 49_1, 72_4, 120_1, 44_1, 159_2, 164_1 and 73_2) and nine down-regulated genes (TDFs \#250_3, 157_2, 11_1, 71_3, 67_4, 10_4, 22_3, 104_1 and 68_2) were isolated from B-toxic C. grandis leaves, while only five up-regulated genes (TDFs \#73_2, 250_3, 157_2, 60_1 and 131_1) and four down-regulated genes (TDFs \#164_1, 71_3, 186_1 and 108_1) were identified in B-toxic C. sinensis leaves. Obviously, B-toxicity affected nucleic acid metabolism more in C. grandis leaves than in C. sinensis 
ones. This agrees with our inference that $C$. sinensis may tolerate higher level of $\mathrm{B}$.

\section{Leaf protein and amino acid metabolism}

All these differentially expressed TDFs encoding chloroplatic translation initiation factor IF-2 (TDF \#236_1) involved in promoting the binding of formylmethionyltRNA to $30 \mathrm{~S}$ ribosomal subunits, eukaryotic release factor 1-3 (TDF \#117_4) involved in the termination step of protein synthesis, EMB1241 (At5g17710; TDF \#93_3 ) related to protein folding and stabilization, Ankyrin repeat domain-containing protein (TDF \#73_3) involved mainly in mediating protein-protein interactions, and ribosomal proteins [i.e., 50S ribosomal protein L15 (TDF \#179_4), 30S ribosomal protein S17 (TDF \#105_1), putative 60S ribosomal protein L6 (TDF \#99_6) and 60S ribosomal protein L4_1 (TDF \#186_2) ] related to mature ribosome assembly and translation processes except for SHEPHERD (TDF \#93_2) involved in the correct folding and/or complex formation of CLAVATA (CLV) proteins [33], 60S ribosomal protein L23 (TDF \#129_2) and 60S ribosomal protein L10B (TDF \#161_1), were down-regulated in B-toxic C. grandis leaves (Table 2), indicating that B-toxicity impairs protein biosynthesis in C. grandis leaves [34,35]. By contrast, only three down-regulated genes [30S ribosomal protein S17 (TDF \#105_1), chaperonin 20 (TDF \#98_1) involved in protein folding and stabilization and AT5G47880 (TDF \#69_3) involved in the termination step of protein synthesis] were detected in B-toxic C. sinensis leaves (Table 2). These results demonstrated that B-toxicity affected protein biosynthesis more in the former than in the latter. This agrees with our data that B-toxicity only decreased total soluble protein concentration in C. grandis leaves (Figure $3 \mathrm{H}$ ).

Here we observed four down-regulated genes [i.e., mitogen-activated protein (MAP) kinase (TDF \#23_4), putative leucine-rich repeat receptor-like protein kinase (TDF \#139-4), CBL-interacting protein kinase 19 (TDF \#72_1) and At1g25390/F2J7_14 (TDF \#39_3)] and three up-regulated genes [i.e., CDK activating kinase (TDF \#12_2), serine/threonine protein kinase ATR (TDF \#22_2) and receptor-like protein kinase (TDF \#235_3) ] involved in phosphorylation and one up-regulated gene [i.e., protein phosphatase $2 \mathrm{C}$ (TDF \#99_1)] involved in dephosphorylation in B-toxic C. grandis leaves, while only one down-regulated gene [i.e., receptor-like protein kinase (TDF \#110_1)] and one up-regulated gene [i.e., protein phosphatase $2 \mathrm{C}$ (TDF \#99_1)] in B-toxic C. sinensis leaves (Table 2). This means that $C$. sinensis leaves might achieve a better balance between phosphorylation and dephosphorylation than C. grandis ones under B-toxicity, which might contribute to the B-tolerance of $C$. sinensis.
Inactive (i.e., incorrect folding) and futile proteins for cell are tagged by ubiquitin for proteolysis [36]. In this study, we found four up-regulated genes [i.e., C3H4 type zinc finger protein (TDF \#99_2), AT5g57360/MSF19_2 (TDF \#54_1), E3 ligase SAP5 (TDF \#57_1) and root phototropism protein 2 (TDF \#234_1)] and three down-regulated genes [i.e., E3 ubiquitin-protein ligase BRE1-like protein (TDF \#96_1), Skp1-like protein 1 (TDF \#187_1) and polyubiquitin (TDF \#120_2)] involved in ubiquitination in B-toxic C. grandis leaves, and one up-regulated gene [i.e., putative E3 ubiquitin-protein ligase XBAT31 isoform 2 (TDF \#158_2)] and two downregulated genes [i.e., F-box family protein (TDF \#73_1) and F-box with WD-40 2 (TDF \#112_1)] involved in ubiquitination in B-toxici $C$. sinensis leaves. This indicates that ubiquitination might be involved in the adaptive response of citrus leaves to B-toxicity. Plant proteases has been shown to play key roles in controlling strict protein quality and degrading specific sets of proteins in response to environmental stresses [37]. As expected, several genes (TDFs \#38_3, 81_1, 38_4, 73_4, 240_1, 39_1, 145_1, 67_1 and 75_1) involved in proteolysis were altered in B-toxic C. grandis and C. sinensis leaves (Table 2).

$\mathrm{S}$-adenosylmethionine (AdoMet) participates in a number of essential metabolic pathways in plants and is the principal biological methyl donor. AdoMet-dependent methylation is essential for keeping cellular functions in plants [38]. Methionine synthase, which catalyzes the last reaction in de novo methionine synthesis, also serves to regenerate the methyl group of AdoMet. As shown in Table 2, B-toxicity increased the expression of AT4G01850 (TDF \#138_4) involved in AdoMet biosynthesis in C. sinensis leaves, but decreased Methionine synthase expression (TDF \#245_1) in C. grandis leaves, which might contribute to the higher tolerance of $C$. sinensis leaves to B-toxicity than that of C. grandis ones.

$\mathrm{N}$-carbamoylputrescine amidase (TDF \#213_4) involved in polyamine (putrescine) biosynthesis were down-regulated in B-toxic C. grandis leaves (Table 2). This means that the biosynthesis of polyamine might be inhibited in B-toxic $C$. grandis leaves, which disagrees with the previous report that $1000 \mu \mathrm{M} \mathrm{B}$ increased leaf concentration of putrescine in B-sensitive barley cultivar, but decreased its concentration in B-tolerant one [39].

The up-regulation of 2-oxoglutarate-dependent dioxygenase gene (TDF \#61_2) in B-toxic C. grandis leaves (Table 2) agrees with the reports that B-toxicity stimulated the general amino acid control system in Saccharomyces cerevisiae [35] and that the concentration of total amino acids in tomato leaves increased under B-toxicity [40]. Evidence shows that 2-oxoglutarate-dependent dioxygenase participates in glucosinolate biosynthesis [41]. Thus, the concentration of glucosinolates might be enhanced in B-toxic C. grandis leaves. 
There is evidence showing that a few cystathionine- $\beta$ synthase (CBS) domain-containing proteins (CDCPs) play a role in plant stress response/tolerance and development [42]. Overexpression of OsCBSX4 improved tobacco plant tolerance to salinity, oxidative, and heavy metal stresses [43]. We observed that B-toxicity decreased the transcript level of CDCP (TDF \#251_3) in C. sinensis leaves (Table 2), as obtained on manganese (Mn)-toxic C. grandis leaves [44]. However, B-deficient C. sinensis roots had higher level of CBS family protein [45]. Singh et al. observed that the expression of OsCBSX4 was up-regulated under high salinity, heavy metal, and oxidative stresses at seedling stage of a salt tolerant (Pokkali) rice cultivar, whilst its expression was upregulated only under $\mathrm{NaCl}$ stress, downregulated under heavy metal stress and kept unchanged under oxidative stress in a salt sensitive (IR64) rice one [43]. Taken all together, the influence of stresses on expression of CDCP genes deponds on the kinds of stresses and plant species/cultivars.

\section{Leaf stress responses}

Inorganic pyrophosphatase (PPase), which cleaves pyrophosphate molecules to liberate two molecules of inorganic phosphate, are essential for the viability of organisms, because the removal of pyrophosphate, a by-product of a host of biosynthetic reactions, is required for preventing the inhibition of thermodynamically unfavorable reactions $[46,47]$. George et al. observed that Nicotiana benthamiana plants lacking plastidial soluble PPase exhibited reduced drought tolerance as a result of the impaired leaf anabolic pathways [46]. The up-regulation of PPase 1 (TDF \#118_1) in B-toxic $C$. sinensis leaves (Table 2) might be an adaptive response to B-toxicity. By contrast, its expression (TDF \#118_1) was down-regulated in B-toxic C. grandis leaves (Table 2).

Because leaf $\mathrm{CO}_{2}$ assimilation was decreased in B-toxic leaves (Figure 3A), less of the absorbed light energy was utilized in photosynthetic electron transport in these leaves, particularly under high light. Thus, reactive oxygen species (ROS) production might be enhanced in B-toxic leaves because of more excess absorbed photon flux [14]. In addition to various ROS scavenger enzymes, "house-keeping" enzymes such as Nudix hydrolases (NUDXs) also play a role in ROS scavenging. Ogawa et al. [48] and Ishikawa et al. [49] showed that transgenic Arabidopsis plants overexpressing AtNUDX2 and AtNUDX7 exhibited higher tolerance to oxidative stress than wild type plants. Therefore, the higher expression level of NUDX19 (TDF \#148_2) in B-toxic C. sinensis leaves might be an adaptive response to B-toxicity (Table 2). However, its expression level (TDF \#148_2) in C. grandis leaves decreased in response to B-toxicity (Table 2).

Up to $10 \%$ of the ascorbate content of the whole leaf is localized in the apoplast, where it forms the first line of defense against external oxidants [50]. In the apoplast, ascorbate oxidase (AO) oxidizes ascorbate to the unstable radical monodehydroascorbate which rapidly disproportionates to yield dehydroascorbate and ascorbate, thus participating in the regulation of the redox state of ascorbic acid pool. AO has been suggested to play a role in cell expansion via the modulation of redox control of the apoplast [51]. Pignocchi et al. [52] showed that enhanced AO activity decreased the concentration and the redox state of ascorbic acid pool in the apoplast, whereas reduced $\mathrm{AO}$ activity increased its amount and redox state in the apoplast. Overexpression of $A O$ in the apoplast of tobacco resulted in lowered capacity for scavenging ROS in the leaf apoplast accompanied by increased sensitivity to ozone [53]. Fotopoulos et al. [50] observed that $A O$-overexpressing transgenic tobacco plants had increased sensitivity to various oxidative stress-promoting agents accompanied by a general suppression of the plant antioxidative metabolism. By contrast, a diminution in $\mathrm{AO}$ activity improved tomato yield under water deficit [54]. The down-regulation of gene encoding Fe (II)/ascorbate oxidase family protein SRG1 (TDF \#59_1) in B-toxic C. sinensis leaves (Table 2) might increase the amount and the redox state of AA pool in the apoplast, thus enhancing the B-tolerance.

Thioredoxins, which participates in supplying reducing power to reductases required for detoxifying lipid hydroperoxides or repairing oxidized proteins, play key roles in plant tolerance of oxidative stress [55]. We found that the expression level of thioredoxin superfamily protein (TDF \#137_2) was up-regulated in B-toxic C. grandis leaves (Table 2), indicating that thioredoxins might be involved in the ROS detoxification. However, the transcript level of thioredoxin superfamily protein (TDF \#68_3) gene was down-regulated in B-toxic C. grandis leaves.

Our finding that B-toxicity increased the expression level of group 5 late embryogenesis abundant protein (LEA5, TDF \#2_1) in C. grandis leaves (Table 2) agrees with the previous report that drought, heat and salt stresses stimulated the expression of LEA5 in citrus leaves [56]. Accumulation of AtRAB28 (LEA5) protein in Arabidopsis through transgenic approach improved the germination rate under standard conditions or salt and osmotic stresses and the cation toxicity tolerance [57]. Also, B-toxicity increased the transcript level of thaumatin-like protein 1 (TLP1, TDF \#125_1) in C. grandis leaves (Table 2). The family of thaumatin-like proteins (also designated PR-5), which comprises proteins with various functions, is induced by biotic and abiotic factors in plants [58]. Therefore, the up-regulation of LEA5 and TLP1 in B-toxic C. grandis leaves might be an adaptive response.

Protein sodium-and lithium-tolerant 1 (SLT1) gene isolated from tobacco (NtSLT1) and A. thaliana (AtSLT1) 
has been implicated in mediating salt tolerance by regulating $\mathrm{Na}^{+}$homeostasis via the calcineurin $(\mathrm{CaN})$ and SPK1/HAL4 (SPK1/HAL4 which encodes a serine-threonine kinase) signal transduction [59]. Later, Antoine et al. [60] showed that rice OsSLT1 had molecular chaperone activity in vitro, and that OsSLT1 could be an important component of the cell immediate defenses against possible protein denaturation and aggregation. The down-regulation of SLT1 (TDF \#99_5) in B-toxic C. grandis leaves (Table 2) means that $\mathrm{Na}^{+}$ homeostasis or related processes mediated by SLT1 are impaired in B-toxic C. grandis leaves.

Plant autophagy plays a role in various stress responses, pathogen defense, and senescence [61]. Xiong et al. [62,63] showed that AtATG18a was necessary for the formation of autophagosomes during nutrient stress and senescence in $A$. thaliana and that autophagy participated in the degradation of oxidized proteins under oxidative stress conditions in Arabidopsis. AtATG18a RNAi plants usually senesce earlier and have lower tolerance to various stresses including drought, salt and oxidative stresses compared with wild-type plants $[61,63]$. Our result showed that the transcript level of transducin/WD40 domain-containing protein (ATG18a, TDF \#104_3) in C. sinensis leaves decreased in response to B-toxicity (Table 2), indicating that autophagy is impaired in $C$. sinensis leaves.

As shown in Table 2, B-toxicity down-regulated the expression of "cold-regulated" gene (cold regulated 314 thylakoid membrane 2, TDF \# 109_1) in C. sinensis leaves and universal stress protein A-like protein (TDF \#150_2) in C. grandis leaves (Table 2), indicating that B-toxicity might affect the tolerance of plants to other stresses.

\section{Leaf signal transduction}

Here four genes involved in signal transduction were altered by B-toxicity (Table 2 and Figure 4). Evidence shows that that signal recognition particle $54 \mathrm{kDa}$ protein (SRP54) plays important roles in chloroplast development $[64,65]$. The down-regulation of signal recognition particle $54 \mathrm{kDa}$ protein 2 (TDF \#182_2) in B-toxic C. grandis leaves (Table 2) means that the biosynthesis of $\mathrm{Chl}$ is impaired in these leaves. This agrees with our results that B-toxicity affected $\mathrm{Chl}$ more in C. grandis leaves than in C. sinensis ones (Figure 3E).

Increasing evidence shows that 14-3-3 proteins play an important role in plant stress responses [66,67]. The most direct evidence for the role of 14-3-3 proteins in stress responses comes from transgenic rice plants over-expressing ZmGF14-6 encoding a maize 14-3-3 protein [68] and cotton plants over-expressing Arabidopsis 14-3-3 $\lambda$ [69]. These transgenic plants displayed enhanced tolerance to drought stress. Heterotrimeric GTP-binding proteins ( $G$ proteins, consisting of subunits $G_{\alpha}, G_{\beta}$, and $G_{\gamma}$ ) are signaling molecules required for various eukaryotic organisms. Joo et al. [70] observed that $A$. thaliana mutant plants losing the $\mathrm{G}_{\beta}$ protein were less tolerant to $\mathrm{O}_{3}$ damage than wild-type plants. Thus, the B-tolerance of $C$. grandis leaves might be down-regulated due to decreased transcript level of genes encoding 14-3-3 protein (TDF \#108_2) and heterotrimeric GTP-binding protein subunit beta 1 (TDF \#200_1) (Table 2).

In higher plants, the endogenous circadian clock is involved in the manipulation of different various cellular processes ranging from photosynthesis to stress responses $[71,72]$. It also confers plants with competitive advantages, including improved photosynthesis, growth and survival [71]. Nakamichi et al. [72] observed that A PRR9, 7 and 5 triple mutant of Arabidopsis had higher tolerance against drought, salt and cold stresses compared to wild type, demonstrating the involvement of the three genes in abiotic stress responses as negative regulators. The up-regulation of pseudo-response regulator 5 (PRR5; TDF \#70_2) in B-toxic C. sinensis leaves (Table 2) agrees with the previous reports that PRR5 was induced by cold treatment in apical shoots of cassava [73] and in Arabidopsis leaves [74]. Fukushima et al. [75] showed that PRR9, 7 and 5 negatively regulated the biosynthetic pathways of Chl, Car, ABA and $\alpha$-tocopherol. This agrees with our results that B-toxici $C$. sinensis leaves had decreased concentrations of $\mathrm{Chl} \mathrm{a}+\mathrm{b}$ and Car (Figure $3 \mathrm{E}$ and $\mathrm{H}$ ).

\section{Leaf cell transport}

As shown in Table 2 and Figure 4, the number of differentially expressed TDFs involved in cell transport was far less in B-toxic C. sinensis leaves than in B-toxic C. grandis ones, meaning that cell transport is less affected in the former than in the latter, which agrees with our inference that $C$. sinensis leaves may tolerate higher level of B.

Most of the differentially expressed TDFs (TDFs \#26_1, 124_3, 66_1, 97_1, 53_1, 210_1, 178_1, 49_3, 137_1, 63_1, 51_1, 250_2, 79_2 and 67_3) associated with cell transport were up-regulated in B-toxic C. grandis leaves except for AT5g24810/F6A4_20 (TDF \#89_2), protein transport protein SEC61 $\gamma$ subunit (TDF \#6_1) and putative $\beta$-subunit of adaptor protein complex 3, PAT2 (TDF \#249_2) (Table 2), indicating that cell transport might be enhanced in B-toxic C. grandis leaves. Plasma-membrane $\mathrm{H}^{+}$-ATPase plays a crucial role in the plant response to environmental stresses, such as salt stress, aluminum $(\mathrm{Al})$ stress, $\mathrm{P}$ and potassium (K) deficiencies [76]. Wu et al. [77] reported that pumping of $\mathrm{Ca}^{2+}$ and $\mathrm{Mn}^{2+}$ by an endoplasmic reticulum-type $\mathrm{Ca}^{2}$ ${ }^{+}$-ATPase (ECA1) into the endoplasmic reticulum was necessary for maintaining plant growth under calcium (Ca)-deficiency or Mn-toxicity. The $\mathrm{P}_{\mathrm{IB}_{\mathrm{B}}}$-ATPases (also known as heavy metal ATPases), which are involved in heavy metal transport across cellular membranes, play a crucial role in metal homeostasis and detoxification in 
plants [78]. Proton pump interactor 1 (PPI1), an interactor of plasma-membrane $\mathrm{H}^{+}$-ATPase, stimulates its activity in vitro [79]. The up-regulation of PPI1 (TDF \#97_1) in B-toxic leaves agrees with our data that the transcript level of $H^{+}$-ATPase 6 (TDF \#26_1) in C. grandis leaves increased in response to B-toxicity (Table 2) and with the report that the expression of PPI1 in potato tuber was up-regulated by salt stress and cold [79].

ATP-binding cassette $(\mathrm{ABC})$ transporters are involved in metal ion efflux from the plasma-membrane. AtPDR8, an $\mathrm{ABC}$ transporter localized in the plasma-membrane of $A$. thaliana root hairs and epidermal cells, confers metal tolerance [80]. Our finding that the expression of ABC transporter G family member 40 (TDF \#53_1) gene was up-regulated in B-toxic $C$. grandis leaves agrees with the reports that AtPDR8 in Arabidopsis roots and shoots was induced when exposed to copper $(\mathrm{Cu})$, cadmium $(\mathrm{Cd})$ and lead $(\mathrm{Pb})$ [80], and that $\mathrm{ABC}$ transporter $\mathrm{G}$ family member 40 gene and $A B C$ transporter A family member 7 gene were induced in drought-sensitive and -tolerant genotypes of Gossypium herbaceum, respectively under drought stress [81]. However, the expression of AT5g24810/F6A4_20 (TDF \#89_2) was down-regulated in B-toxic C. grandis leaves (Table 2).

$\mathrm{Cu}$ transporters (COPTs/Ctrs) are involved in the maintenance of $\mathrm{Cu}$ homeostasis in plants. Generally speaking, COPTs/Ctrs are up-regulated by $\mathrm{Cu}$ deprivation and down-regulated by $\mathrm{Cu}$ excess [82]. COPT1 antisense Arabidopsis plants have decreased $\mathrm{Cu}$ level due to decreased $\mathrm{Cu}$ uptake and display sensitivity to $\mathrm{Cu}$ chelators [83]. The up-regulation of COPT (TDF \#210_1) in B-toxic C. grandis leaves might play a role in the maintenance of leaf $\mathrm{Cu}$ homeostasis.

Plant cyclic nucleotide gated channels (CNGCs) paly a role in heavy metal homeostasis. Previous study showed that transgenic tobacco plants overexpressing a truncated NtCBP4 (tobacco $C N G C$ ) had higher tolerance to $\mathrm{Pb}$ compared with wild type [84]. Chan et al. [85] reported that $\mathrm{cng}_{\mathrm{g}} 2$ Arabidopsis mutants were hypersensitive to increased soil $\mathrm{Ca}$. However, transgenic tobacco plants overexpressing $N t C B P 4$ were hypersensitivity to $\mathrm{Pb}$ [86]. B-toxicity-induced increase in transcript level of CNGC1 (TDF \#178_1) in C. grandis leaves (Table 2) agrees with the report that the expression of AtCNGC2 was induced during Arabidopsis leaf senescence and AtCNGC2 might be involved in programmed cell death [87].

Membrane traffic is not only required for plant normal cellular function and maintenance of cellular viability, but also plays an important roles in plant responses to the environment $[88,89]$. The transcript levels of genes [i.e., vacuolar-sorting receptor 3 (TDF \#49_3), vacuolar protein-sorting-associated protein 37-1 (TDF \#137_1), vesicle-associated membrane protein-associated protein (TDF \#63_1), secY protein transport family protein
(TDF \#51-1), fat-free-like protein (TDF \#250_2) and non-specific lipid-transfer protein (TDF \#79_2)] involved in membrane traffic increased in B-toxic C. grandis leaves except for genes encoding protein transport protein SEC61 $\gamma$ subunit (TDF \#6_1) and putative $\beta$-subunit of adaptor protein complex 3, PAT2 (TDF \# 249_2) (Table 2). This indicates that membrane traffic might be enhanced in B-toxic C. grandis leaves.

Plant sieve element occlusion (SEO) genes have been shown to encode the common phloem proteins (P-proteins) that plug sieve plates after wounding. Tobacco SEO-RNA interference lines were essentially devoid of P-protein structures and lost photoassimilates more rapidly after injury than control plants [90]. Therefore, the up-regulation of sieve element occlusion protein 1 gene (TDF \#67_3) in B-toxic C. grandis leaves (Table 2) might be of advantage to prevent the loss of photoassimilates. Recently, Huang et al. observed that many electron-dense particles deposited near sieve plates of B-toxic C. grandis and C. sinensis leaves [13]. In conclusion, the up-regulation of cell transport in B-toxic C. grandis leaves might be an adaptive response of plants to B-toxicity.

By contrast, we isolated three down-regulated [i.e., putative $\beta$-subunit of adaptor protein complex 3, PAT2 (TDF \#249_2), sugar transporter ERD6-like 5 (TDF \#61_1) and metal tolerance protein (MTP, TDF \#179_2)] and three up-regulated [i.e., sieve element occlusion protein 1 (TDF \#67_3), kinesin-related protein (TDF \#51_4) and bidirectional sugar transporter SWEET7 (TDF \#36_2) TDFs from B-toxic C. sinensis leaves (Table 2). Generally speaking, cell transport might be not enhanced in B-toxicity leaves.

In plants, kinesins are involved in a variety of cellular processes including intracellular transport, spindle assembly, phragmoplast assembly, chromosome motility, MAP kinase regulation and microtubule stability [91]. $\mathrm{Li}$ et al. [92] reported that mutation of rice $\mathrm{BC12}$ / GDD1 encoding a kinesin-like protein led to dwarfism with impaired cell elongation. Nishihama et al. [93] demonstrated that the expansion of the cell plate in tobacco plant cytokinesis required kinesin-like proteins (i.e., NACK1 and NACK2) to regulate the activity and localization of MAP kinase kinase kinase. Therefore, the up-regulation of kinesin-like protein (TDF \#51_4) in $C$. sinensis leaves (Table 2) might be an adaptive responsive to B-toxicity. However, the transcript level of putative $\beta$-subunit of adaptor protein complex 3, PAT2 (TDF \#249_2) in C. sinensis leaves decreased in response to B-toxicity (Table 2 ).

Plant SWEETs function as facilitators involved in the influx and the efflux of sugar into and out of cells [94]. We found that the expression level of SWEET7 (TDF \#36_2) in $C$. sinensis leaves increased in response to B-toxicity 
(Table 2), which agrees with the previous report that SWEET15/SAG29 was enhanced in senescing Arabidopsis leaves [95]. However, the expression of gene encoding sugar transporter ERD6-like 5 (TDF \#61_1), a passive facilitator for the diffusion of glucose across the tonoplast membrane, was down-regulated in B-toxic C. sinensis leaves (Table 2). This disagrees with the previous report that the expression of AtESL1 (ERD six-like 1) was induced by various stresses including drought, high salinity and ABA in Arabidopsis plants [96].

MTPs are a subfamily of the cation diffusion facilitator (CDF) family found in plants. So far, most studied CDF family members confer heavy metal tolerance by affecting heavy metal efflux from the cytoplasm [97]. The down-regulation of MTP (TDF \#179_2) in C. sinensis leaves (Table 2) means that the tolerance of plants to heavy metal might be reduced in B-toxic plants. This agrees with our previous report that the tolerance of $C$. grandis plants to Al-toxicity was higher under adequate B supply than under excess B [98].

\section{Leaf cell wall and cytoskeleton modification}

Eleven TDFs associated with cell wall and cytoskeleton modification were altered by B-toxicity (Table 2 and Figure 4). O-methyltransferase (OMT) genes are involved in lignin biosynthesis. Fu et al. [99] showed that down-regulation of the caffeic acid 3-O-methyltransferase (COMT) gene in switchgrass lowered lignin level in whole tillers and stems of transgenic plants and enhanced forage quality. Transgenic Leucaena leucocephala plants expressing antisense OMT displayed decreased activity of OMT activity and concentration of lignin [100]. Therefore, the biosynthesis of lignin in B-toxic C. grandis and C. sinensis leaves might be reduced due to decreased expression of COMT (TDF \#49_4) and COMT3 (TDF \#125_2) (Table 2). In addition, the biosynthesis of chitin in $C$. grandis and $C$. sinensis leaves and cellulose in $C$. grandis leaves might be down-regulated under B-toxicity due to the downregulation of chitinase (TDF \#10_3) and cellulose synthase (TDF \#249_4) (Table 2). These results demonstrated that B-toxicity might impair citrus cell wall metabolism, which agrees with the previous suggestion that leaf cupping, a specific visible B-toxic symptom in some species might be due to the inhibition of cell wall expansion, through disturbance of cell wall crosslinks [101]. However, the transcript levels of genes encoding OMT1 (TDF \#33_3), LIM domain-containing protein (TDF \#241_1), UDP-glucose flavonoid 7-O-glucosyltransferase (TDF \#124_2), UDPglucosyltransferase family 1 protein (TDF \#3_3), limonoid UDP-glucosyltransferase (TDF \#70_4) and putative glucosyltransferase (TDF \#63_2) in C. grandis increased in response to B-toxicity (Table 2).

Evidence shows that lily LIM1 [87] and all Arabidopsis LIM domain proteins [102] participate in regulating actin cytoskeleton organization and dynamics. Tobacco LIM1 protein acts in the cytoplasm as an actin binding and bundling protein [103] and in the nucleus as a transcription factor regulating the expression of genes related to lignin biosynthesis [104]. Recently, Moes et al. [105] demonstrated the involvement of tobacco LIM2 in actin-bundling and histone gene transcription. The up-regulation of LIM domain-containing protein (TDF \#241_1) in B-toxic C. grandis leaves (Table 2) agrees with the report that the expression of LIM domaincontaining protein in Physcomitrella patens increased under cold acclimation [106].

Glycosyltransferases (GTs), which catalyze the formation of glycosidic bonds between donor sugars and acceptor molecules, participate in many aspects of a plant life, including cell wall biosynthesis [107,108]. In Arabidopsis, up to 10 or 12 GT2 family members form the cellulose synthase catalytic subunit and callose synthase gene families [108]. In plants, UDP-glucosyltransferases (UGTs) have been suggested to play important roles in keeping cell homeostasis, regulating plant growth and improving their tolerance to environmental stresses [109]. Overexpression of UGT74E2 conferred tolerance to salinity and drought stresses in A. thaliana [110]. Transgenic tobacco plants overexpressiong UGT85A5 exhibited enhanced salt tolerance [111]. Therefore, the up-regulation of UDP-glucose flavonoid 7-O-glucosyltransferase (TDF \#124_2), UGT family 1 protein (TDF \#3_3), limonoid UGT (TDF \#70_4) and putative GT (TDF \#63_2) genes in B-toxic C. grandis leaves (Table 2) might play a role in B-tolerance of plants. However, loss of function of a UGT73B2 alone or in conjunction with $U G T 73 B 1$ and $U G T 73 B 3$ resulted in enhanced oxidative stress tolerance in Arabidopsis, whilst transgenic Arabidopsis plants overexpressing UGT73B2 displayed decreased oxidative stress tolerance [112].

\section{Others}

Overexpression of bacterial or plant gene encoding phytoene synthase (PSY), a key regulatory enzyme in Car biosynthesis, led to enhanced level of total Car in various higher plants [113,114]. Transgenic Arabidopsis plants overexpressing PSY from euhalophyte Salicornia europaea had higher tolerance to salt stress than wild type plants by enhanced photosynthetic efficiency and antioxidative capacity [115]. Cidade et al. [116] showed that ectopic expression of PSY from Citrus paradisi fruit conferred abiotic stress tolerance in transgenic tobacco, which was correlated with the increased endogenous ABA level and expression of stress-responsive genes. Our finding that B-toxic C. grandis leaves had lower transcript of PSY (TDF \#229_4; Table 2) means that the biosynthesis of Car and the antioxidative capacity may be decreased in B-toxic leaves. This agrees with our data that B-toxicity affected Car more in C. grandis leaves than in C. sinensis 
one (Figure 3G) and the inference that C. grandis may tolerate lower level of $B$.

Strictosidine synthase (Str), a key enzyme in alkaloid biosynthesis, catalyzes the condensation of tryptamine and secologanin leading to the synthesis of numerous monoterpenoid indole alkaloids in higher plants [117]. The up-regulation of Str family protein gene (TDF \#231_1) in B-toxic C. sinensis leaves (Table 2) agrees with the previous report that Str in Catharanthus roseus leaves was enhanced under dehydration, salt and UV stresses [117] and that B-toxicity decreased IAA level in Triticum durum seedlings [118], because the expression of Str was inhibited by auxin [119]. B-toxicity-induced up-regulation of Str family protein gene (TDF \#231_1) also agrees with our reference that the AOS pathway and jasmonate level might be up-regulated in the B-toxic $C$. sinensis leaves due to decreased expression of fatty acid HPL gene (TDF \#10_1) (Table 2), because Str has been shown to be induced by jasmonate [120]. By contrast, the expression of Str family protein gene (TDF \#231_1) was down-regulated in B-toxic C. grandis leaves (Table 2), which agrees with the previous report that cold stress led to Str down-regulation in C. roseus leaves [117].

\section{Conclusions}

B-toxicity affected C. grandis seedling growth, leaf $\mathrm{CO}_{2}$ assimilation, pigments, total soluble protein, MDA and $\mathrm{P}$ more than $C$. sinensis, indicating that $C$. sinensis have higher B-tolerance than $C$. grandis ones. Under B-toxicity, C. sinensis leaves accumulated more B than C. grandis ones, meaning that the former may tolerate higher level of B. Using cDNA-AFLP, we successfully isolated 67 up-regulated and 65 down-regulated TDFs from B-toxic C. grandis leaves, whilst only 31 up-regulated and 37 down-regulated TDFs from B-toxic C. sinensis ones. This indicates that gene expression is less affected in B-toxic C. sinensis leaves than in C. grandis ones, which might be associated with the fact that $C$. sinensis leaves can tolerate higher level of $\mathrm{B}$. The higher B-tolerance of $C$. sinensis might be related to the findings that B-toxic $C$. sinensis leaves had higher expression levels of genes involved in photosynthesis, which might contribute to the higher photosynthesis and light utilization and less excess light energy compared to the B-toxic C. grandis ones, and in ROS scavenging, thus preventing them from photo-oxidative damage. In addition, B-toxicity-induced alteration in the expression levels of genes encoding inorganic PPase 1, AT4G01850 and methionine synthase differed between the two species, which might also contribute to the B-tolerance of $C$. sinensis. In this study, a total of 174 differentially expressed TDFs were isolated from two citrus species, only $26 \mathrm{TDFs}$ presented in the two citrus, the remaining TDFs presented only in C. grandis or C. sinensis, demonstrating that the
B-toxicity-responsive genes differ between the two citrus species. For example, cell transport were up-regulated in B-toxicity C. grandis leaves, whilst this did not occur in B-toxic C. sinensis ones.

\section{Methods}

\section{Plant materials}

This study was conducted from February to December, 2011 at Fujian Agriculture and Forestry University. Plant culture and B treatments were performed according to Han et al. [14]. Briefly, 5-week-old uniform seedlings of 'Xuegan' (Citrus sinensis) and 'Sour pummelo' (Citrus grandis) were transplanted to $6 \mathrm{~L}$ pots containing fine river sand. Plants, two per pot, were grown in a greenhouse under natural photoperiod at Fujian Agriculture and Forestry University. Eight weeks after transplanting, each pot was supplied every other day until dripping with nutrient solution containing $10 \mu \mathrm{M}$ (control) or $400 \mu \mathrm{M}$ (B-toxic) $\mathrm{H}_{3} \mathrm{BO}_{3}$ and $6 \mathrm{mM} \mathrm{KNO}, 4 \mathrm{mM} \mathrm{Ca}\left(\mathrm{NO}_{3}\right)_{2}$, $2 \mathrm{mM} \mathrm{NH}_{4} \mathrm{H}_{2} \mathrm{PO}_{4}, 1 \mathrm{mM} \mathrm{MgSO}, 10 \mu \mathrm{M} \mathrm{H}_{3} \mathrm{BO}_{3}, 2 \mu \mathrm{M}$ $\mathrm{MnCl}_{2}, 2 \mu \mathrm{M} \mathrm{ZnSO}_{4}, 0.5 \mu \mathrm{M} \mathrm{CuSO}_{4}, 0.065 \mu \mathrm{M}\left(\mathrm{NH}_{4}\right)$ ${ }_{6} \mathrm{Mo}_{7} \mathrm{O}_{24}$ and $20 \mu \mathrm{M}$ Fe-EDTA for 15 weeks. At the end of the experiment, fully expanded leaves from different replicates and treatments were used for all the measurements. Leaves were collected at noon under full sun and immediately frozen in liquid nitrogen and were stored at $-80^{\circ} \mathrm{C}$ until extraction.

\section{Measurements of plant DW, root and leaf $B$, leaf $P$, total soluble protein, MDA and pigments}

Ten plants per treatment from different pots were harvested and divided into their parts (roots and shoots). The plant parts were then dried at $75^{\circ} \mathrm{C}$ for $48 \mathrm{~h}$ and their DWs measured. B concentration in roots and leaves was assayed by ICP emission spectrometry after microwave digestion with $\mathrm{HNO}_{3}$ [121]. Leaf P concentration was measured according to Ames [122]. Leaf total soluble protein was measured according to Bradford [123] using bovine serum albumin as standard after being extracted with $50 \mathrm{mM} \mathrm{Na} \mathrm{HPO}_{4}-\mathrm{KH}_{2} \mathrm{PO}_{4}(\mathrm{pH}$ 7.0) and $5 \%(\mathrm{w} / \mathrm{v})$ insoluble polyvinylpyrrolidone. Extraction and determination of leaf MDA were performed according to Hodges et al. [124]. Chl, Chl a, Chl b and Car were assayed according to Lichtenthaler [125] after being extracted with $80(\mathrm{v} / \mathrm{v})$ actetone.

\section{Measurements of leaf gas exchange}

Leaf gas exchange was measured using a CIARS-2 portable photosynthesis system (PP systems, Herts, UK) at ambient $\mathrm{CO}_{2}$ concentration under a controlled light intensity of 990-1010 $\mu \mathrm{mol} \mathrm{m}^{-2} \mathrm{~s}^{-1}$ between 9:00 and 11:00 on a clear day. During measuring, leaf temperature and air relative humidity were $32.2 \pm 0.2^{\circ} \mathrm{C}$ and $66.6 \pm 0.8 \%$, respectively. 


\section{Leaf RNA extraction, CDNA synthesis and CDNA-AFLP analysis}

Total RNA was extracted from ca. $300 \mathrm{mg}$ of frozen mixed leaves from B-toxic and control plants of $C$. grandis and C. sinensis using Recalcirtant Plant Total RNA Extraction Kit (Centrifugal column type, Bioteke Corporation, China). There were three biological replicates for each treatment. Leave of 4-5 plants from different pots were mixed as a biological replicate. Equal amounts of leaves were collected from each plant. cDNA synthesis and cDNA-AFLP analysis were performed according to Zhou et al. [44].

\section{Validation of CDNA-AFLP data using qRT-PCR}

Total RNA was extracted from the frozen leaves as described above. qRT-PCR analysis was performed according to Zhou et al. [44]. Specific primers were designed from the sequences of 16 differentially expressed TDFs using Primer Primier Version 5.0 (PREMIER Biosoft International, CA, USA). The sequences of the $\mathrm{F}$ and $\mathrm{R}$ primers used were listed in Additional file 3. Samples for qRT-PCR were run in 3 biological replicates with 3 technical replicates. Leave of $4-5$ plants from different pots were mixed as a biological replicate. Relative gene expression was calculated using ddCt algorithm. For the normalization of gene expression, citrus actin (GU911361.1) was used as an internal standard and the leaves from control plants were used as reference sample, which was set to 1 .

\section{Experimental design and statistical analysis}

There were 20 pots ( 40 seedlings) per treatment in a completely randomized design. Experiments were performed with 3-10 replicates. Results represented the mean \pm SE. Statistical analyses of data were carried out by ANOVA tests. Means were separated by the least significant difference test at $P<0.05$ level.

\section{Additional files}

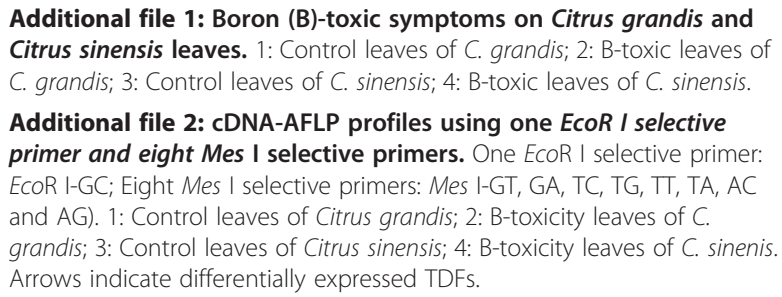

Additional file 3: Specific primer pairs used for qRT-PCR expression analysis.

\section{Abbreviations}

ABC: ATP-binding cassette; ACP: Acyl carrier protein; AdoMet: Sadenosylmethionine; AO: Ascorbate oxidase; AOS: Allene oxide synthase; B: Boron; Car: carotenoid; CBS: Cystathionine- $\beta$-synthase; CDCP: CBS domaincontaining protein; CDF: Cation diffusion facilitator; CDNA-AFLP: CDNA- amplified fragment length polymorphism; Chl: Chlorophyll; CNGC: Cyclic nucleotide gated channel; COMT: Caffeic acid 3-O-methyltransferase; COPT: Cu transporters; DW: Dry weight; GT: Glycosyltransferase; HPL: Hydroperoxide lyase; IF: Initiation factor; LEA5: Group 5 late embryogenesis abundant protein; MAP: Mitogen-activated protein; MATE: Multi-drug and toxic compound extrusion; MDA: Malondialdehyde; MTP: Metal tolerance protein; NUDX: Nudix hydrolases; OMT: O-methyltransferase; POR: Protochlorophyllide oxidoreductase; PPase: Pyrophosphatase; PPI1: Proton pump interactor 1; PRR: Pseudo-response regulator 5; PsbA: PSII 32 kDa protein; PsbP: PSII 23 kDa protein; PSI: Photosystem I; PSII: Photosystem II; PSY: Phytoene synthase; RNAi: RNA interference; ROS: Reactive oxygen species; Rubisco: RuBP carboxylase/oxygenase; RuBP: ribulose-1,5-bisphosphate;

SBPase: Sedoheptulose-1,7-bisphosphatase; SEO: Sieve element occlusion; SLT1: Protein sodium-and lithium-tolerant 1; Str: Strictosidine synthase; TDF: Transcript-derived fragments; TLP: Thaumatin-like protein; UGT: UDP-glucosyltransferase.

\section{Competing interests}

The authors declare that they have no competing interests.

\section{Authors' contributions}

PG carried out most of the experiments and drafted the manuscript. YPQ participated in the design of the study. LTY participated in the design of the study and coordination. XY carried out the measurement of B and P. HXJ performed the statistical analysis. JHH carried out the cultivation of seedlings. LSC designed and directed the study and revised the manuscript. All authors have read and approved the final manuscript.

\section{Acknowledgement}

This study was financially supported by the earmarked fund for China Agriculture Research System.

\section{Author details}

${ }^{1}$ College of Resource and Environmental Science, Fujian Agriculture and Forestry University, Fuzhou 350002, China. ${ }^{2}$ Institute of Horticultural Plant Physiology, Biochemistry and Molecular Biology, Fujian Agriculture and Forestry University, Fuzhou 350002, China. ${ }^{3}$ Institute of Materia Medica, Fujian Academy of Medical Sciences, Fuzhou 350001, China. ${ }^{4}$ College of Life Science, Fujian Agriculture and Forestry University, Fuzhou 350002, China. ${ }^{5}$ Institute of Fruit Tree Science, Fujian Academy of Agricultural Sciences, Fuzhou 350013, China. ${ }^{6}$ Fujian Key Laboratory for Plant Molecular and Cell Biology, Fujian Agriculture and Forestry University, Fuzhou 350002, China. ${ }^{7}$ The Higher Educational Key Laboratory of Fujian Province for Soil Ecosystem Health and Regulation, Fujian Agriculture and Forestry University, Fuzhou 350002, China.

Received: 13 September 2014 Accepted: 14 October 2014 Published online: 28 October 2014

References

1. Nable RO, Banuelos GS, Paull JG: Boron toxicity. Plant Soil 1997, 193:181-198.

2. Papadakis IE, Dimassi KN, Therios IN: Response of two citrus genotypes to six boron concentrations: concentration and distribution of nutrients, total absorption, and nutrient use efficiency. Aust J Agr Res 2003, 54:571-580.

3. Cervilla LM, Blasco B, Ríos J, Romero L, Ruiz J: Oxidative stress and antioxidants in tomato (Solanum lycopericum) plants subjected to boron toxicity. Ann Bot 2007, 100:747-756.

4. Parks $J \mathrm{~L}$, Edwards M: Boron in the environment. Crit Rev Environ Sci Technol 2005, 35:81-114.

5. Kasajima I, Fujiwara T: Identification of novel Arabidopsis thaliana genes which are induced by high levels of boron. Plant Biotech 2007, 24:355-360

6. Hassan M, Oldach K, Baumann U, Langridge P, Sutton T: Genes mapping to boron tolerance QTL in barley identified by suppression subtractive hybridization. Plant Cell Environ 2010, 33:188-198.

7. Aquea F, Federici F, Moscoso C, Vega A, Jullian P, Haseloff J, Arce-Johnson P: A molecular framework for the inhibition of Arabidopsis root growth in response to boron toxicity. Plant Cell Environ 2012, 35:719-734. 
8. Chen $L S$, Han S, Qi YP, Yang LT: Boron stresses and tolerance in citrus. Afr J Biotech 2012, 11:5961-5969.

9. Sheng O, Zhou GF, Wei QJ, Peng SA, Deng XX: Effects of excess boron on growth, gas exchange, and boron status of four orange scion-rootstock combinations. J Plant Nutr Soil Sci 2010, 173:469-476.

10. Huang YZ, Li J, Wu SH, Pang DM: Nutrition condition of the orchards in the main production areas of Guanxihoney pomelo trees (Pinhe county). J Fujian Agri Univ 2001, 30:40-43.

11. Papadakis IE, Dimassi KN, Bosabalidis AM, Therios IN, Patakas A, Giannakoula A: Effects of $B$ excess on some physiological and anatomical parameters of 'Navelina' orange plants grafted on two rootstocks. Environ Exp Bot 2004, 51:247-257.

12. Papadakis IE, Dimassi KN, Bosabalidis AM, Therios IN, Patakas A, Giannakoula A. Boron toxicity in 'Clementine' mandarin plants grafted on two rootstocks. Plant Sci 2004, 166:539-547.

13. Huang JH, Cai ZJ, Wen SX, Guo P, Ye X, Lin GZ, Chen LS: Effects of boron toxicity on root and leaf anatomy in two citrus species differing in boron tolerance. Trees Struct Funct 2014, doi:10.1007/s00468-014-1075-1.

14. Han S, Tang N, Jiang HX, Yang LT, Li Y, Chen LS: $\mathrm{CO}_{2}$ assimilation, photosystem II photochemistry, carbohydrate metabolism and antioxidant system of citrus leaves in response to boron stress. Plant $\mathrm{SC}$ 2009, 176:143-153

15. Sheng O, Song SW, Chen YJ, Peng SA, Deng XX: Effects of exogenous B supply on growth, $B$ accumulation and distribution of two navel orange cultivars. Trees Struct Funct 2009, 23:59-68.

16. Hudson GS, Evans JR, von Caemmerer S, Arvidsson YBC, Andrewset TJ: Reduction of ribulose-1,5-bisphosphate carboxylase/oxygenase content by antisense RNA reduces photosynthesis in transgenic tobacco plants. Plant Physiol 1992, 98:294-302.

17. Khan MS, Hameed W, Nozoe M, Shiina T: Disruption of the psbA gene by the copy correction mechanism reveals that the expression of plastid-encoded genes is regulated by photosynthesis activity. I Plant Res 2007, 120:421-430

18. Ishihara S, Yamamoto Y, Ifuku K, Sato F: Functional analysis of four members of the PsbP family in photosystem II in Nicotiana tabacum using differential RNA interference. Plant Cell Physiol 2005, 46:1885-1893.

19. Ifuku K, Yamamoto Y, Ono TA, Ishihara S, Sato F: PsbP protein, but not PsbQ protein, is essential for the regulation and stabilization of photosystem II in higher plants. Plant Physiol 2005, 139:1175-1184.

20. Yabe T, Morimoto K, Kikuchi S, Nishio K, Terashima I, Nakai M: The Arabidopsis chloroplastic NifU-Like protein $\mathrm{CnfU}$, which can act as an iron-sulfur cluster scaffold protein, is required for biogenesis of ferredoxin and photosystem I. Plant Cell 2004, 16:993-1007.

21. Price GD, Evans JR, Voncaemmerer S, Yu JW, Badger MR: Specific reduction of chloroplast glyceraldehyde-3-phosphate dehydrogenase-activity by antisense RNA reduces $\mathrm{CO}_{2}$ assimilation via a reduction in ribulose-bisphosphate regeneration in transgenic tobacco plants. Planta 1995, 195:369-378.

22. Harrison EP, Olcer H, Lloyd JC, Long SP, Raines CA: Small decreases in SBPase cause a linear decline in the apparent RuBP regeneration rate, but do not affect Rubsico carboxylation capacity. J Exp Bot 2001, 52:1779-1784.

23. Lefebvre S, Lawson T, Fryer M, Zakhleniuk OV, Lloyd JC, Raines CA: Increased sedoheptulose-1,7-bisphosphatase activity in transgenic tobacco plants stimulates photosynthesis and growth from an early stage in development. Plant Physiol 2005, 138:451-460.

24. Wang ML: Molecular Cloning and Transformation of Sedoheptulose-1,7Bisphosphatase in Lycopersicon esculentum, PhD thesis. China: Shandong Agricultural University; 2011.

25. Baud $S$, Wuillème $S$, Dubreuca B, de Almeida A, Vuagnat $C$, Lepiniec $L$, Miquel $\mathrm{M}$, Rochat $\mathrm{C}$ : Function of plastidial pyruvate kinases in seeds of Arabidopsis thaliana. Plant J 2007, 52:405-419.

26. Frick G, Su Q, Apel K, Armstrong GA: An Arabidopsis porB porC double mutant lacking light-dependent ADPH:protochlorophyllide oxidoreductases $\mathrm{B}$ and $\mathrm{C}$ is highly chlorophyll-deficient and developmentally arrested. Plant J 2003, 35:141-153.

27. Pattanayak GK, Tripathy BC: Overexpression of protochlorophyllide oxidoreductase $\mathrm{C}$ regulates oxidative stress in Arabidopsis. PLoS One 2011, 6:e26532.

28. Gorinova N, Nedkovska M, Atanassov A: Cytochrome P450 monooxygenases as a tool for metabolization herbicides in plants. Biotechnol Biotechnol Eq 2005, 19(Special issue):105-115.
29. Narusaka Y, Narusaka M, Seki M, Umezawa T, Ishida J, Nakajima M, Enju A, Shinozaki K: Crosstalk in the responses to abiotic and biotic stresses in Arabidopsis: analysis of gene expression in cytochrome P450 gene superfamily by cDNA microarray. Plant Mol Biol 2004, 55:327-342.

30. Liu X, Li F, Tang J, Wang W, Zhang F, Wang G, Chu J, Yan C, Wang T, Chu C, Li C: Activation of the jasmonic acid pathway by depletion of the hydroperoxide lyase OSHPL3 reveals crosstalk between the HPL and AOS branches of the oxylipin pathway in rice. PLoS One 2012, 7:e50089.

31. Tang GY, Wei LQ, Liu ZJ, Bi YP, Shan L: Ectopic expression of peanut acyl carrier protein in tobacco alters fatty acid composition in the leaf and resistance to cold stress. Biol Plant 2012, 56:493-501.

32. Branen JK, Shintani DK, Engeseth NJ: Expression of antisense acyl carrier protein-4 reduces lipid content in Arabidopsis leaf tissue. Plant Physiol 2003, 132:748-756.

33. Ishiguro S, Watanabe $Y$, Ito N, Nonaka H, Takeda N, Sakai T, Kanaya H, Okada K: SHEPHERD is the Arabidopsis GRP94 responsible for the formation of functional CLAVATA proteins. EMBO J 2002, 21:898-908.

34. Chen M, Mishra S, Heckathorn SA, Frantz JM, Krause C: Proteomic analysis of Arabidopsis thaliana leaves in response to acute boron deficiency and toxicity reveals effects on photosynthesis, carbohydrate metabolism, and protein synthesis. J Plant Physiol 2014, 171:235-242.

35. Uluisik I, Alaattin Kaya A, Fomenko DE, Karakaya HC, Carlson BA, Gladyshev VN, Koc A: Boron stress activates the general amino acid control mechanism and inhibits protein synthesis. PLOS One 2012, 6:e27772.

36. Mazzucotelli E, Mastrangelo AM, Crosatti C, Guerra D, Stanca AM, Cattivelli L: Abiotic stress response in plants: when post-transcriptional and post-translational regulations control transcription. Plant Sci 2008 174:420-431.

37. García-Lorenzo M, Sjödin A, Jansson S, Funk C: Protease gene families in Populus and Arabidopsis. BMC Plant Biol 2006, 6:30.

38. Sauter M, Moffatt $B$, Saechao MC, Hell R, Wirtz M: Methionine salvage and S-adenosylmethionine: essential links between sulfur, ethylene and polyamine biosynthesis. Biochem J 2013, 451:145-154

39. Roessner $U$, Patterson JH, Forbes $M G$, Fincher $G B$, Langridge $P$, Bacic $A$ : An investigation of boron toxicity in barley using metabolomics. Plant Physiol 2006, 142:1087-1101.

40. Cervilla LM, Blasco B, Ríos JJ, Rosales MA, Rubio-Wilhelmi MM, Sánchez-Rodríguez E, Romero L, Ruiz JM: Response of nitrogen metabolism to boron toxicity in tomato plants. Plant Biol 2009, 11:671-677.

41. Baskar V, Gururani MA, Yu JW, Park SW: Engineering glucosinolates in plants: current knowledge and potential uses. Appl Biochem Biotechnol 2012, 168:1694-1717.

42. Kushwaha HR, Singh AK, Sopory SK, Singla-Pareek SL, Pareek A: Genome wide expression analysis of CBS domain containing proteins in Arabidopsis thaliana (L.) Heynh and Oryza sativa L. reveals their developmental and stress regulation. BMC Genomics 2009, 10:200

43. Singh AK, Kumar R, Pareek A, Sopory SK, Singla-Pareek SL: Overexpression of rice CBS domain containing protein improves salinity, oxidative, and heavy metal tolerance in transgenic tobacco. Mol Biotechnol 2012, 52:205-216.

44. Zhou CP, Qi YP, You X, Yang LT, Guo P, Ye X, Zhou XX, Ke FJ, Chen LS: Leaf CDNA-AFLP analysis of two citrus species differing in manganese tolerance in response to long-term manganese-toxicity. BMC Genomics 2013, 14:621.

45. Yang LT, Qi YP, Lu YB, Guo P, Sang W, Feng H, Zhang HX, Chen LS: iTRAQ protein profile analysis of Citrus sinensis roots in response to long-term boron-deficiency. J Proteomics 2013, 93:179-206.

46. George GM, van der Merwe MJ, Nunes-Nesi A, Bauer R, Fernie AR, Kossmann J, Lloyd JR: Virus-induced gene silencing of plastidial soluble inorganic pyrophosphatase impairs essential leaf anabolic pathways and reduces drought stress tolerance in Nicotiana benthamiana. Plant Physiol 2010, 154:55-66.

47. Hernández-Domíguez EE, Valencia-Turcotte LG, Rodríguez-Sotres R: Changes in expression of soluble inorganic pyrophosphatases of Phaseolus vulgaris under phosphate starvation. Plant Sci 2012, 187:39-48.

48. Ogawa T, Ishikawa K, Harada K, Fukusaki E, Yoshimura K, Shigeoka S: Overexpression of an ADP-ribose pyrophosphatase, AtNUDX2, confers enhanced tolerance to oxidative stress in Arabidopsis plants. Plant J 2009, 57:289-301

49. Ishikawa K, Ogawa T, Hirosue E, Nakayama Y, Harada K, Fukusaki E, Yoshimura K, Shigeoka S: Modulation of the poly (ADP-ribosyl) ation 
reaction via the Arabidopsis ADP-ribose/NADH pyrophosphohydrolase, AtNUDX7, is involved in the response to oxidative stress. Plant Physiol 2009, 151:741-754.

50. Fotopoulos V, Sanmartin M, Kanellis AK: Effect of ascorbate oxidase over-expression on ascorbate recycling gene expression in response to agents imposing oxidative stress. J Exp Bot 2006, 57:3933-3943.

51. Kato N, Esaka M: Expansion of transgenic tobacco protoplasts expressing pumpkin ascorbate oxidase is more rapid that that of wild type protoplasts. Planta 2000, 210:1018-1022.

52. Pignocchi C, Kiddle G, Hernández I, Foster SJ, Asensi A, Taybi T, Barnes J, Foyer $\mathrm{CH}$ : Ascorbate oxidase-dependent changes in the redox state of the apoplast modulate gene transcript accumulation leading to modified hormone signaling and orchestration of defense processes in tobacco. Plant Physiol 2006, 141:423-435.

53. Sanmartin M, Drogoudi PA, Lyons T, Pateraki I, Barnes J, Kanellis AK: Over-expression of ascorbate oxidase in the apoplast of transgenic tobacco results in altered ascorbate and glutathione redox states and increased sensitivity to ozone. Planta 2003, 216:918-928.

54. Garchery C, Gest N, Do PT, Alhagdow M, Baldet P, Menard G, Rothan C, Massot C, Gautier H, Aarrouf J, Fernie AR, Stevens R: A diminution in ascorbate oxidase activity affects carbon allocation and improves yield in tomato under water deficit. Plant Cell Environ 2013, 36:159-175.

55. Vieira Dos Santos C, Rey P: Plant thioredoxins are key actors in the oxidative stress response. Trends Plant Sci 2006, 11:329-334.

56. Naot D, Ben-Hayyim G, Eshdat Y, Holland D: Drought, heat and salt stress induce the expression of a citrus homologue of an atypical late-embryogenesis Lea5 gene. Plant Mol Biol 1995, 27:619-622.

57. Borrell A, Cutanda MC, Lumbreras V, Pujal J, Goday A, Culiáñez-Macià FA, Pagès M: Arabidopsis thaliana atrab28: a nuclear targeted protein related to germination and toxic cation tolerance. Plant Mol Biol 2002, 50:249-259.

58. Ahmed NU, Park Jl, Jung HJ, Chung MY, Cho YG, Nou IS: Characterization of thaumatin-like gene family and identification of Pectobacterium carotovorum subsp. carotovorum inducible genes in Brassica oleracea. Plant Breed Biotech 2013, 1:111-121.

59. Matsumoto TK, Pardo JM, Takeda S, Bressan RA, Hasegawa PM: Tobacco and Arabidiopsis SLT1 mediate salt tolerance of yeast. Plant Mol Biol 2001, 45:489-500.

60. Antoine W, Stewart JM, Reyes BG D I: The rice homolog of the sodium/ lithium tolerance (SLT1) gene functions as molecular chaperone in vitro. Physiol Plant 2005, 125:299-310.

61. Liu Y, Bassham DC: Autophagy: pathways for self-eating in plant cells. Annu Rev Plant Biol 2012, 63:215-237.

62. Xiong $Y$, Contento AL, Bassham DC: AtATG18a is required for the formation of autophagosomes during nutrient stress and senescence in Arabidopsis thaliana. Plant J 2005, 42:535-546.

63. Xiong Y, Contento AL, Nguyen PQ, Bassham DC: Degradation of oxidized proteins by autophagy during oxidative stress in Arabidopsis. Plant Physiol 2007, 143:291-299.

64. Richter CV, Bals T, Schünemann D: Component interactions, regulation and mechanisms of chloroplast signal recognition particle-dependent protein transport. Eur J Cell Biol 2010, 89:965-973.

65. Zhang F, Luo X, Hu B, Yong Wan Y, Xie J: YGL138(t), encoding a putative signal recognition particle $54 \mathrm{kDa}$ protein, is involved in chloroplast development of rice. Rice 2013, 6:7

66. Chevalier D, Morris ER, Walker JC: 14-3-3 and FHA domains mediate phosphoprotein interactions. Annu Rev Plant Biol 2009, 60:67-91.

67. Chen F, Li Q, Sun L, He Z: The rice 14-3-3 gene family and its involvement in responses to biotic and abiotic stress. DNA Res 2006, 13:53-63.

68. Campo S, Peris-Peris C, Montesinos L, Peñas G, Messeguer J, San Segundo B: Expression of the maize ZmGF14-6 gene in rice confers tolerance to drought stress while enhancing susceptibility to pathogen infection. J Exp Bot 2012, 63:983-999.

69. Yan J, He C, Wang J, Mao Z, Holaday SA, Allen RD, Zhang H: Overexpression of the Arabidopsis 14-3-3 protein GF14 $\lambda$ in cotton leads to a "stay-green" phenotype and improves stress tolerance under moderate drought conditions. Plant Cell Physiol 2004, 45:1007-1014.

70. Joo JH, Wang SY, Chen JG, Jones AM, Fedoroff NV: Different signaling and cell death roles of heterotrimeric $G$ protein $a$ and $b$ subunits in the Arabidopsis oxidative stress response to ozone. Plant Cell 2005, 17:957-970
71. Dodd AN, Salathia N, Hall A, Kévei E, Tóth R, Nagy F, Hibberd JM, Millar AJ, Webb AA: Plant circadian clocks increase photosynthesis, growth, survival, and competitive advantage. Science 2005, 309:630-633.

72. Nakamichi N, Kusano M, Fukushima A, Kita M, Ito S, Yamashino T, Saito K, Sakakibara H, Mizuno T: Transcript profiling of an Arabidopsis PSEUDO RESPONSE REGULATOR arrhythmic triple mutant reveals a role for the circadian clock in cold stress response. Plant Cell Physiol 2009, 50:447-462.

73. An D, Yang J, Zhang P: Transcriptome profiling of low temperature-treated cassava apical shoots showed dynamic responses of tropical plant to cold stress. BMC Genomics 2012, 13:64.

74. Byun YJ, Kim HJ, Lee DH: LongSAGE analysis of the early response to cold stress in Arabidopsis leaf. Planta 2009, 229:1181-1200.

75. Fukushima A, Kusano M, Nakamichi N, Kobayashi M, Hayashi N, Sakakibara H, Mizuno T, Saito K: Impact of clock-associated Arabidopsis pseudo-response regulators in metabolic coordination. Proc Natl Acad Sci USA 2009, 106:7251-7256.

76. Shen $H$, Chen J, Wang Z, Yang C, Sasaki T, Yamamoto Y, Matsumoto $H$, Yan X: Root plasma membrane $\mathrm{H}^{+}$-ATPase is involved in the adaptation of soybean to phosphorus starvation. J Exp Bot 2006, 57:1353-1362.

77. Wu Z, Liang F, Hong B, Young JC, Sussman MR, Harper JF, Sze H: An endoplasmic reticulum-bound $\mathrm{Ca}^{2+} / \mathrm{Mn}^{2+}$ pump, ECA1, supports plant growth and confers tolerance to $\mathrm{Mn}^{2+}$ stress. Plant Physiol 2002, 130:128-137.

78. Takahashi R, Bashir K, Ishimaru Y, Nishizawa NK, Nakanishi H: The role of heavy-metal ATPases, HMAs, in zinc and cadmium transport in rice. Plant Signal Behav 2012, 7:1605-1607.

79. García MNM, País SM, Téllez-Iñón MT, Capiati DA: Characterization of StPPI1, a proton pump interactor from Solanum tuberosum L. that is up-regulated during tuber development and by abiotic stress. Planta 2011, 233:661-674.

80. Kim DY, Bovet $L$, Maeshima M, Martinoia E, Lee $Y$ : The $A B C$ transporter AtPDR8 is a cadmium extrusion pump conferring heavy metal resistance. Plant J 2007, 50:207-218.

81. Ranjan A, Pandey N, Lakhwani D, Dubey NK, Pathre UV, Sawant SV Comparative transcriptomic analysis of roots of contrasting Gossypium herbaceum genotypes revealing adaptation to drought. BMC Genomics 2012, 13:680.

82. Yuan M, Li X, Xiao J, Wang S: Molecular and functional analyses of COPT/Ctr-type copper transporter-like gene family in rice. BMC Plant Biol 2011, 11:69.

83. Sancenón V, Puig S, Mateu-Andrés I, Dorcey E, Thiele DJ, Peñarrubia L: The Arabidopsis copper transporter COPT1 functions in root elongation and pollen development. J Biol Chem 2004, 279:15348-15355.

84. Sunkar R, Kaplan B, Bouche N, Arazi T, Dolev D, Talke I, Maathuis FJM, Sanders D, Bouchez D, Fromm H: Expression of a truncated tobacco NtCBP4 channel in transgenic plants and disruption of the homologous Arabidopsis CNGC1 gene confer $\mathrm{Pb}^{2+}$ tolerance. Plant J 2000, 24:533-542.

85. Chan CW, Schorrak LM, Smith RK Jr, Bent AF, Sussman MR: A cyclic nucleotide-gated ion channel, CNGC2, is crucial for plant development and adaptation to calcium stress. Plant Physiol 2003, 132:728-731.

86. Arazi $T$, Sunkar R, Kaplan B, Fromm H: A tobacco plasma membrane calmodulin-binding transporter confers $\mathrm{Ni}^{2+}$ tolerance and $\mathrm{Pb}^{2+}$ hypersensitivity in transgenic plants. Plant J 1999, 20:171-182.

87. Köhler C, Merkle T, Roby D, Neuhaus G: Developmentally regulated expression of a cyclic nucleotide-gated ion channel from Arabidopsis indicates its involvement in programmed cell death. Planta 2001, 213:327-332.

88. Ohno H: Membrane traffic in multicellular systems: more than just a housekeeper. J Biochem 2006, 139:941-942.

89. Wang HJ, Wan AR, Jauh GY: An actin-binding protein, LILIM1, mediates calcium and hydrogen regulation of actin dynamics in pollen tubes. Plant Physiol 2008, 147:1619-1636.

90. Ernst AM, Jekat SB, Zielonka S, Müller B, Neumann U, Rüping B, Twyman RM, Krzyzanek V, Prüfer D, Noll GA: Sieve element occlusion (SEO) genes encode structural phloem proteins involved in wound sealing of the phloem. Proc Natl Acad Sci USA 2012, 109:E1980-E1989.

91. Shen Z, Collatos AR, Bibeau JP, Furt F, Vidali L: Phylogenetic analysis of the kinesin superfamily from Physcomitrella. Front Plant Sci 2012, 3:230.

92. Li J, Jiang J, Qian Q, Xu Y, Zhang C, Xiao J, Du C, Luo W, Zou G, Chen M, Huang $Y$, Feng $Y$, Cheng Z, Yuan M, Chong K: Mutation of rice BC12/GDD1, which encodes a kinesin-like protein that binds to a GA biosynthesis 
gene promoter, leads to dwarfism with impaired cell elongation. Plant Cell 2011, 23:628-640.

93. Nishihama R, Soyano T, Ishikawa M, Araki S, Tanaka H, Asada T, Irie K, Ito M, Terada M, Banno H, Yamazaki Y, Machida Y: Expansion of the cell plate in plant cytokinesis requires a kinesin-like protein/MAPKKK complex. Cell 2002, 109:87-99.

94. Slewinski TL: Diverse functional roles of monosaccharide transporters and their homologs in vascular plants: a physiological perspective. Mol Plant 2011, 4:641-662

95. Quirino BF, Reiter WD, Amasino RD: One of two tandem Arabidopsis genes homologous to monosaccharide transporters is senescence-associated. Plant Mol Biol 1999, 46:447-457.

96. Yamada K, Osakabe Y, Mizoi J, Nakashima K, Fujita Y, Shinozaki K, Yamaguchi-Shinozaki K: Functional analysis of an Arabidopsis thaliana abiotic stress-inducible facilitated diffusion transporter for monosaccharides. J Biol Chem 2010, 285:1138-1146.

97. Ricachenevsky FK, Menguer PK, Sperotto RA, Williams LE, Fett JP: Roles of plant metal tolerance proteins (MTP) in metal storage and potential use in biofortification strategies. Front Plant Sci 2013, 4:144.

98. Jiang HX, Tang N, Zheng JG, Chen LS: Antagonistic actions of boron against inhibitory effects of aluminum toxicity on growth, $\mathrm{CO}_{2}$ assimilation, ribulose-1,5-bisphosphate carboxylase/oxygenase, and photosynthetic electron transport probed by the JIP-test, of Citrus grandis seedlings. BMC Plant Biol 2009, 9:102.

99. Fu C, Mielenz JR, Xiao X, Ge Y, Hamilton CY, Rodriguez M Jr, Chen F, Foston M, Ragauskas A, Bouton J, Dixon RA, Wang ZY: Genetic manipulation of lignin reduces recalcitrance and improves ethanol production from switchgrass. Proc Natl Acad Sci USA 2011, 108:3803-3808.

100. Rastogi S, Dwivedi UN: Down-regulation of lignin biosynthesis in transgenic Leucaena leucocephala harboring O-methyltransferase gene. Biotechnol Prog 2006, 22:609-916.

101. Loomis WD, Durst RW: Chemistry and biology of boron. Biofactors 1992 , 3:229-239.

102. Papuga J, Hoffmann C, Dieterle M, Moes D, Moreau F, Tholl S, Steinmetz A Thomas C: Arabidopsis LIM proteins: a family of actin bundlers with distinct expression patterns and modes of regulation. Plant Cell 2010, 22:3034-3052

103. Thomas C, Hoffmann C, Dieterle M, Van Troys M, Ampe C, Steinmetz A: Tobacco WLIM1 is a novel F-Actin binding protein involved in actin cytoskeleton remodeling. Plant Cell 2006, 18:2194-2206.

104. Kawaoka A, Kaothien P, Yoshida K, Endo S, Yamada K, Ebinuma H: Functional analysis of tobacco LIM protein Ntlim1 involved in lignin biosynthesis. Plant J 2000, 22:289-301.

105. Moes D, Gatti S, Hoffmann C, Dieterle M, Moreau F, Neumann K, Schumacher M, Diederich M, Grill E, Shen WH, Steinmetz A, Thomas C: A LIM domain protein from tobacco involved in actin-bundling and histone gene transcription. Mol Plant 2013, 6:483-502.

106. Sun MM, Li LH, Xie H, Ma RC, He YK: Differentially expressed genes under cold acclimation in Physcomitrella patens. J Biochem Mol Biol 2007 40:986-1001.

107. Harholt J, Sørensen I, Fangel J, Roberts A, Willats WGT, Scheller HV, Petersen BL, Banks JA, Ulvskov P: The glycosyltransferase repertoire of the spikemoss Selaginella moellendorffii and a comparative study of its cell wall. PLoS One 2012, 7:e35846.

108. Scheible WR, Pauly M: Glycosyltransferases and cell wall biosynthesis: novel players and insights. Curr Opin Plant Biol 2004, 7:285-295.

109. Bowles D, Lim EK, Poppenberger B, Vaistij FE: Glycosyltransferases of lipophilic small molecules. Annu Rev Plant Biol 2006, 57:567-597.

110. Tognetti VB, Van Aken O, Morreel K, Vandenbroucke K, van de Cotte B, De Clercq I, Chiwocha S, Fenske R, Prinsen E, Boerjan W, Genty B, Stubbs KA, Inzé D, Van Breusegem F: Perturbation of indole-3-butyric acid homeostasis by the UDP-glucosyltransferase UGT74E2 modulates Arabidopsis architecture and water stress tolerance. Plant Cell 2010, 22:2660-2679.

111. Sun YG, Wang B, Jin SH, Qu XX, Li YJ, Hou BK: Ectopic expression of Arabidopsis glycosyltransferase UGT85A5 enhances salt stress tolerance in tobacco. PloS One 2013, 8:e59924.

112. Kim IA, Heo JO, Chang KS, Lee SA, Lee MH, Lim CE, Lim J: Overexpression and inactivation of UGT73B2 modulate tolerance to oxidative stress in Arabidopsis. J Plant Biol 2010, 53:233-239.

113. Rodríguez-Villalón A, Gas E, Rodríguez-Concepción M: Phytoene synthase activity controls the biosynthesis of carotenoids and the supply of their metabolic precursors in dark-grown Arabidopsis seedlings. Plant J 2009, 60:424-435.

114. Zhang J, Tao N, Xu Q, Zhou W, Cao H, Xu J, Deng X: Functional characterization of citrus PSY gene in Hongkong kumquat (Fortunella hindsii Swingle). Plant Cell Rep 2009, 28:1737-1746.

115. Han H, Li Y, Zhou S: Overexpression of phytoene synthase gene from Salicornia europaea alters response to reactive oxygen species under salt stress in transgenic Arabidopsis. Biotechnol Lett 2008, 30:1501-1507.

116. Cidade LC, de Oliveira TM, Mendes AFS, Macedo AF, Floh EIS, Gesteira AS, Soares-Filho WS, Costa MGC: Ectopic expression of a fruit phytoene synthase from Citrus paradisi Macf. promotes abiotic stress tolerance in transgenic tobacco. Mol Biol Rep 2012, 39:10201-10209.

117. Dutta A, Sen J, Deswal R: New evidences about strictosidine synthase (Str) regulation by salinity, cold stress and nitric oxide in Catharanthus roseus. J Plant Biochem Biotech 2013, 22:124-131.

118. Gemici M, Aktaş LY, Türkyilmaz B, Güven A: The effects of the excessive boron applications on indole-3-acetic acid levels in Triticum durum Desf cv. Gediz seedlings. Cumhuriyet Üniversitesi Fen Bilimleri Dergisi 2002, 23(2):17-24.

119. Pasquali G, Goddijn OJ, de Waal A, Verpoorte R, Schilperoort RA, Hoge JH, Memelink J: Coordinated regulation of two indole alkaloid biosynthetic genes from Catharanthus roseus by auxin and elicitors. Plant Mol Biol 1992, 18:1121-1131.

120. Menke FL, Champion A, Kijne JW, Memelink J: A novel jasmonate- and elicitor-responsive element in the periwinkle secondary metabolite biosynthetic gene Str interacts with a jasmonate- and elicitor-inducible AP2-domain transcription factor, ORCA2. EMBO J 1999, 18:4455-4463.

121. Wang J, Nakazato T, Kinya Sakanishi K, Yamada O, Tao H, Saito I: Single-step microwave digestion with $\mathrm{HNO}_{3}$ alone for determination of trace elements in coal by ICP spectrometry. Talanta 2006, 68:1584-1590.

122. Ames BN: Assay of inorganic phosphate, total phosphate and phosphatase. Methods Enzymol 1966, 8:115-118.

123. Bradford MM: A rapid and sensitive method for quantitation of microgram quantities of protein utilizing the principle of protein-dye binding. Anal Biochem 1976, 72:248-254.

124. Hodges DM, DeLong JM, Forney CF, Prange RK: Improving the thiobarbituric acid-reactive-substances assay for estimating lipid peroxidation in plant tissues containing anthocyanin and other interfering compounds. Planta 1999, 207:604-611.

125. Lichtenthaler HK: Chlorophylls and carotenoids: pigments of photosynthetic biomembranes. Methods Enzymol 1987, 148:350-382.

doi:10.1186/s12870-014-0284-5

Cite this article as: Guo et al:: CDNA-AFLP analysis reveals the adaptive responses of citrus to long-term boron-toxicity. BMC Plant Biology 2014 14:284

\section{Submit your next manuscript to BioMed Central and take full advantage of:}

- Convenient online submission

- Thorough peer review

- No space constraints or color figure charges

- Immediate publication on acceptance

- Inclusion in PubMed, CAS, Scopus and Google Scholar

- Research which is freely available for redistribution 